\title{
Meromorphic solutions of algebraic differential equations
}

\section{A.E. Eremenko}

\section{CONTENTS}

Introduction

§1. Algebraic function fields and the Fuchs conditions 63

§2. Admissible solutions $\quad 69$

§3. Proof of Theorem 5 73

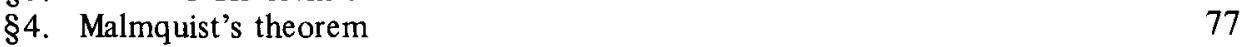

$\S 5$. The asymptotic behaviour of solutions $\quad 80$

§6. Higher-order equations $\quad 83$

\$7. Autonomous equations of Briot-Bouquet type 88 References

\section{Introduction}

We talk of an algebraic differential equation if it is of the form

$$
F\left(y^{(k)}, y^{(k-1)}, \ldots, y, z\right)=0, \quad y^{(j)}=\frac{d^{j} y}{d z^{j}},
$$

where $F$ is a polynomial in the first $k+1$ variables, whose coefficients are analytic functions of the independent variable $z$. If the conditions of Cauchy's theorem for the existence and uniqueness of the solution are satisfied, then (0.1) determines an analytic function in a neighbourhood of a given point $z_{0}$. One of the most difficult problems in the analytic theory of differential equations is that of the analytic continuation of the solution and of studying it in the whole domain where it exists. It is natural, first of all, to ask whether there are solutions of $(0.1)$ that are meromorphic in the finite plane C. A second important problem is to study the properties of meromorphic solutions immediately from the equation, if it is known that such solutions exist. This article is devoted to this second problem. Throughout what follows, unless stated otherwise, by a meromorphic function we mean one that is meromorphic in $\mathbf{C}$. We always use $z$ to denote an independent variable, $z \in \mathbf{C}$.

For autonomous first-order equations
$F\left(y^{\prime}, y\right)=0$ 
both questions were completely solved in the 19th century. A necessary and sufficient condition for the existence of meromorphic solutions is given by the Fuchs criterion (see $\$ 1$ ). Every meromorphic solution of $(0.2)$ is either an elliptic function, or a rational function, or a function of the form $R(\exp a z)$, where $R$ is rational and $a \in \mathrm{C}$ (see $\S 7$ ).

The first result on meromorphic solutions of non-autonomous equations was the famous theorem of Malmquist [41]: if the equation $y^{\prime}=R(y, z)$, where $R$ is a rational function of two variables, admits a transcendental meromorphic solution, then this equation necessarily is of the form $y^{\prime}=a(z) y^{2}+b(z) y+c(z)$, that is, it is a Riccati equation. Malmquist's theorem has many times been generalized and proved by different methods (see, for example, [3], Ch. V; [22], §5; [31], Ch. IV, and also [32] and [69]). The simplest proof due to Künzi [37] is by the use of the Nevanlinna theory of meromorphic functions. Starting from the papers of Yosida [69], Künzi [37], and Wittich [62], [63], Nevanlinna theory became a fundamental tool for the relevant questions.

Many papers have been devoted to generalizations of Malmquist's theorem to first-order equations that are non-linear in $y^{\prime}$. As long ago as 1920 , Malmquist proved the following general theorem [42]: if a first-order equation

$$
F\left(y^{\prime}, y, z\right)=0,
$$

where $F$ is a polynomial in the first two variables with algebraic coefficients, is irreducible and has a transcendental meromorphic solution, then this equation satisfies the Fuchs conditions (see also [43]). For brevity, we call Malmquist's theorems of 1913 and 1920 Theorems I and II, respectively. It is easy to see that Theorem I is a special case of II.

The paper [42] has had practically no influence on the work of other authors, although many results obtained after 1920 are simple consequences of Theorem II. A typical example is [55], in which Malmquist's Theorem II in a weakened form is suggested as a conjecture. Apparently this situation is explained by the fact that [42] is written very laconically, and many essential points of the proofs in it are omitted. It is possible that Malmquist's Theorem II has aroused distrust. It should be mentioned that for an understanding of [42] and [43] the review by Schmidt [53] is of great help.

Up to a short time ago, all the results on meromorphic solutions of $(0.1)$ have been obtained under the assumption that the coefficients of $F$ are algebraic or rational functions of $z$. It was thanks to the approach proposed independently by Laine [38] and A.E. and V.D. Mokhon'ko [14] that it became possible to study meromorphic solutions of equations with transcendental coefficients. This approach consists, roughly speaking, in considering "admissible solutions", that is, those whose Nevanlinna characteristic grows faster than the characteristics of the coefficients of the equation. If the latter are algebraic, then the class of admissible solutions 
coincides with that of transcendental solutions (see $\S 2$ ). This accords well with the classical point of view that one should consider solutions of differential equations in a class of more "complicated" functions than the coefficients of the equation (see, for example, [2], 171; [4], 37). In the case in question, the growth of the Nevanlinna characteristic is a good "measure of transcendence" of an algebroid or meromorphic function.

If $F$ is a polynomial in three variables, then $(0.3)$ can be regarded as the equation of an algebraic surface $F\left(t_{1}, t_{2}, t_{3}\right)=0$. Another point of view turns out to be more fruitful if $F$ in $(0.3)$ is regarded as a polynomial in two variables over some suitable coefficient field. This approach allows one to apply the theory of algebraic functions in one variable to $(0.3)$, where the algebraic dependence of $F$ on $z$ becomes unimportant. In this article we show how the consistent application of this idea allows us to obtain almost all the most recent results on admissible solutions for equations of both the first and higher orders.

In $\S \S 1$ and 2 we gather all the information about algebraic function fields, Fuchsian differential fields, and the Nevanlinna theory that is necessary for what follows. In $\S \S 3$ and 4 we give a new proof and a generalization of Malmquist's Theorem II, and also a survey of recent results on the existence of meromorphic solutions of (0.3). In $\S 5$ we investigate, with the aid of the theory set out in $\S \S 1-4$, the asymptotic properties of solutions of (0.3) in the most interesting case, when the dependence of $F$ on $z$ is algebraic. $\S 6$ is devoted to analogues of the results of $\S \S 4$ and 5 for equations of higher order, and also for solutions that are meromorphic in a circle or a half-plane. Finally, in $\S 7$ we examine higher-order equations of the special form $F\left(y^{(k)}, y\right)=0$ with constant coefficients (equations of Briot-Bouquet type). We have tried not to repeat results in the books [1], $[2],[3],[4],[27]$, and [31].

The author is sincerely grateful to A.A. Gol'dberg, who drew his attention to the classical papers of Malmquist, proposed this article and gave comprehensive help in writing it out, and also to V.E. Katsnel'son and V.G. Drinfel'd for useful discussions.

\section{$\S 1$. Algebraic function fields and the Fuchs conditions}

1. It is convenient to regard the coefficients of $(0.1)$ as elements of some algebraically closed field. We choose the field $A$ of all algebroid functions, that is, the algebraic closure of the field of meromorphic functions. $A$ can be described in the following way. We consider first the field of formal series of the form

$$
\sum_{j=0}^{\infty} a_{j} z^{(m+j) / n}, \quad a_{j} \in \mathbf{C}, \quad a_{0} \neq 0, \quad m \in \mathbf{Z}, \quad n \in \mathbf{N} .
$$

This field is algebraically closed. We always assume that the exponents of the powers in (1.1) of terms with $a_{j} \neq 0$ do not all have a common factor. 
We now pick out those series (1.1) that converge in some neighbourhood of $\mathbf{O}$ and can be continued in $\mathbf{C}$ to a finitely-valued analytic function without singularities in $\mathbf{C}$ other than algebraic ones. These series also form an algebraically closed field, which, as is not hard to see, is the algebraic closure $A$ of the field of meromorphic functions. By an algebroid function we always mean an element of $A$, that is, a series (1.1) continued in C. To two distinct elements of $A$ there may correspond identical analytic functions in the usual sense of the word, for example, $z^{1 / 2}$ and $-z^{1 / 2}$ are, by our definition, distinct algebroid functions.

We shall have to deal with inequalities of the form

$$
\Phi\left(f_{1}(z), f_{2}(z), \ldots, f_{n}(z)\right) \geqslant 0, \quad z \in \mathbf{C}, f_{j} \in A .
$$

Such inequalities must be interpreted in the following way. We consider power series of the form $(1.1)$, representing elements $f_{1}, \ldots, f_{n}$. The germs corresponding to these series can be analytically continued along a curve $\Gamma$ to a point $z \in \mathbf{C}$, and the curve is the same for all the germs and does not pass through the branch points of $f_{1}, \ldots, f_{n}$. The result of the continuation must satisfy (1.2) irrespective of the choice of $\Gamma$. An equation containing $f(z), f \in A, z \in \mathbf{C}$, is to be interpreted in a similar way.

2. Let $A_{0}$ be a field. We always assume, without specifically saying so, that $A_{0}$ is of characteristic 0 and algebraically closed. As a rule, $A_{0}$ will be an algebraically closed subfield of $A$. We use the usual notation $A_{0}\left[t_{1}, \ldots, t_{n}\right]$ and $A_{0}\left(t_{1}, \ldots, t_{n}\right)$, respectively, for the ring of polynomials and the field of rational functions in $n$ variables with coefficients in $A_{0}$. We also use the notation $B\left[t_{1}, \ldots, t_{n}\right]$ when $B$ is a ring.

Before examining differential equations, we must study relations of the form $F(x, y)=0$, where $F \in A_{0}\left[t_{1}, t_{2}\right]$. Suppose that $F \in A_{0}\left[t_{1}, t_{2}\right]$ is an irreducible polynomial. Then the quotient $\operatorname{ring} A_{0}\left[t_{1}, t_{2}\right] /(F)$, where $(F)$ is the ideal generated by $F$, is an integral domain, and we can consider its field of fractions $\mathfrak{Y}$, which for brevity we denote by $\mathfrak{A}=A_{0}\left(t_{1}, t_{2}\right) / F$. The elements of $\mathfrak{O}$ must be representable as rational functions in $A_{0}\left(t_{1}, t_{2}\right)$ whose numerator and denominator are relatively prime to $F$. The pair $\left(\%, A_{0}\right)$ of the form indicated is called an algebraic function field in a single variable over $A_{0}$. Since $A_{0}$ is fixed, we take the liberty of just calling $\mathfrak{I}$ an algebraic function field.

If $A_{0}=\mathrm{C}$, then $\mathfrak{O}$ is the field of rational functions on the Riemann surface of the function $y$ given by the irreducible equation $F(y, z)=0$, $F \in \mathbf{C}\left[t_{1}, t_{2}\right]$. Information about algebraic fields that we need later is contained in [11], Ch. 1, [20], and [21] (for the case $A_{0}=\mathbf{C}$, see, for example, [9] ).

The field $\mathfrak{A}$ has transcendence degree 1 over $A_{0}$. This means that for any $\alpha, \beta \in \mathscr{Q}$ i there is a polynomial $Q \in A_{0}\left[t_{1}, t_{2}\right]$ for which $Q(\alpha, \beta)=0$.

A ring $V \subset \mathfrak{A}$ is called a local ring if $A_{0} \subset V, \mathfrak{A} \neq V$, and for every either $\alpha \in V$ or $\alpha^{-1} \in V$. The elements $\alpha \in \mathfrak{A}$ such that both $\alpha \in V$ and 
$\alpha^{-1} \in V$ are called units of $V$. The set of all units in $V$ is denoted by $U(V)$. The elements of a local ring $V$ that are not units form an ideal $I=I(V)$. This ideal is maximal and has a single generator $t$. Every element $\alpha \in V$ can be represented in the form $\alpha=t^{m} u, u \in U(V), m \in \mathbf{Z}^{+}$. $\mathfrak{A}$ is the field of fractions of any of its local rings, therefore, each element $\alpha \in \mathscr{U}$ can be represented in any local ring in the form

$$
\alpha=t^{m} u, \quad u \in U(V), \quad m \in \mathbf{Z},
$$

where $m \geqslant 0$ if and only if $\alpha \in V$ in (1.3). The number $m$ in (1.3) is called the order of $\alpha$ in $V$ and is denoted by ord $V \alpha$.

It can be shown that the quotient ring $V / I$ is the same as the field $A_{0}$. Therefore, any element $\alpha \in V$ can be represented in the form $\alpha=a_{0}+\alpha_{1}$, $a_{0} \in A_{0}, \alpha_{1} \in I$, consequently, $\alpha_{1}=t \alpha_{2}, \alpha_{2} \in V$. Continuing this procedure we obtain a formal expansion of $\alpha \in V$ in a Puiseux series

$$
\alpha=t^{m} a_{0}+t^{m+1} a_{1}+\ldots, a_{j} \in A_{0} .
$$

Here $m \geqslant 0$. A similar expansion can also be obtained for any element $\alpha \in \mathfrak{U}$, making use of (1.3). Here $m=\operatorname{ord}_{V} \alpha$ in (1.4) can be any integer.

When $A_{0}=\mathbf{C}$, a local ring is the set of all rational functions on the Riemann surface that do not have a pole at a given point. Thus, local rings are in one-to-one correspondence with the points of the Riemann surface. The ideal $I$ of a local ring is the set of rational functions that vanish at the point in question. As the generator $t$ of $I$ one can take any function that has a simple zero at the given point. This terminology can naturally also be used in the case of an arbitrary field $A_{0}$. We sometimes refer to local rings as points, and to a generator of $I$ as a local parameter at the point. If $m>0$ in (1.3), we say that the element $\alpha$ has a zero of order $m$ at the point $V$, and if $m<0$, that it has a pole of order $-m$. In the case of an arbitrary field $A_{0}$, just as for $A_{0}=\mathrm{C}$, an arbitrary element $\alpha \in \mathfrak{A}$ has $\operatorname{ord}_{V} \alpha \neq 0$ at only finitely many points $V$, and $\sum \operatorname{ord}_{V} \alpha=0$, where the summation is over all points of $\mathfrak{Q}$.

3. A divisor is a point function that takes integer values and is different from 0 at only finitely many points. The sum of all the values taken by a divisor $\delta$ is called its degree and is written deg $\delta$. The divisor $\delta(\alpha)$ of an element $\alpha \in \mathfrak{A}$ is the point function equal to $\operatorname{ord}_{V} \alpha$ at $V$. Here $\operatorname{deg} \delta(\alpha)=0$ for any $\alpha \in \mathfrak{A}$. A divisor is called non-negative if all its values are nonnegative. The divisors form an Abelian group under addition, which is isomorphic to the free Abelian group generated by the points. We use the expression $\delta_{1} \geqslant \delta_{2}$ if $\delta_{1}-\delta_{2} \geqslant 0$ for divisors $\delta_{1}$ and $\delta_{2}$, that is, $\delta_{1}-\delta_{2}$ is a non-negative divisor.

Let $\delta$ be a fixed divisor. We consider the set $\mathcal{L}(\delta)$ of the elements of with the property that $\delta(\alpha) \geqslant-\delta$. It is easy to see that $\mathcal{L}(\delta)$ is a vector space over $A_{0}$. The following theorem enables us to compute the dimension of $\mathcal{L}(\delta)$. 
The Riemann-Roch theorem. There is an integer $g \geqslant 0$, depending only on the fields $A_{0}$ and $\mathfrak{A}$, such that for any divisor

$$
\operatorname{dim} \mathcal{L}(\delta)=\operatorname{deg} \delta+1-g+\Delta(\delta),
$$

where $\Delta(\delta) \geqslant 0$, and $\Delta(\delta)=0$ if $\operatorname{deg} \delta \geqslant 2 g-1$.

Here $\operatorname{dim} \mathcal{L}<0$ means that $\mathcal{L}$ is empty. The number $g$ is called the genus of 2 over $A_{\mathbf{0}}$.

4. As we have already said, any two elements $\alpha, \beta \in \mathfrak{Q}$ are connected by a relation

$$
Q(\alpha, \beta)=Q_{m}(\alpha) \beta^{m}+\ldots+Q_{0}(\alpha)=0, Q_{j} \in A_{0}\left[t_{1}\right] .
$$

We wish to find a connection between ord ${ }_{V} \alpha$ and $\operatorname{ord}_{V} \beta$ for those points $V$ of $\mathscr{U}$ at which $\operatorname{ord}_{V} \alpha<0$. The following classical method serves to do this. On the plane we plot the points with the coordinates $\left(j, \operatorname{deg} Q_{j}\right), 0 \leqslant j \leqslant m$, and two more points $(0,0)$ and $(m, 0)$. We consider the polygon that is the convex hull of this set of points. From the boundary of the polygon we remove the vertical segments and the segment $[0, m]$ of the $j$-axis. The remaining part of the boundary is called the Newton diagram of the polynomial (1.5).

The gradients of the Newton diagram, taken with the opposite sign, form a finite set of rational numbers. This set coincides exactly with the set of numbers $\operatorname{ord}_{V} \beta / \operatorname{ord}_{V} \alpha$, where $V$ ranges over the finite set of points at which $\operatorname{ord}_{V} \alpha<0$.

We also need the following well-known fact. Suppose that the polynomial (1.5) is irreducible. For the existence of a point $V$ such that ord $\alpha \geqslant 0$ and $\operatorname{ord}_{V} \beta<0$ it is necessary and sufficient that $\operatorname{deg} Q_{m}>0$. More precisely,

$$
\operatorname{deg} Q_{m}=-\Sigma \operatorname{ord}_{V} \beta,
$$

where the summation is over all points $V$ such that $\operatorname{ord}_{V} \alpha \geqslant 0$ and $\operatorname{ord}_{V} \beta<0$.

5. The theory of first-order differential equations can be constructed formally, without using the notion of a solution. To do this we must consider, instead of a single equation, the collection of all equations that are obtained from each other by a rational change of the unknown function and its derivative. We give the precise definitions.

Let $\mathfrak{A}=A_{0}\left(t_{1}, t_{2}\right) / F$ be an algebraic function field in one variable over $A_{0}$. An operator $D: \mathfrak{A} \rightarrow \mathfrak{A}$ is called a differentiation if it has the following properties: $D\left(A_{0}\right) \subset A_{0}, D(\alpha+\beta)=D(\alpha)+D(\beta), D(\alpha \beta)=\alpha D \beta+\beta D \alpha$.

The pair $(\mathscr{A}, D)$ (more precisely, the triple $\left.\left(\mathfrak{A}, A_{0}, D\right)\right)$ is called a differential algebraic function field in one variable over the base field $A_{0}$.

Suppose that the action of $D$ is given on $A_{0}$. In order to extend $D$ to the whole field $\mathfrak{U}$ it is sufficient to specify the action of $D$ on one element $\alpha \in \mathscr{R} \backslash A_{0}$, and this can be done arbitrarily. For suppose that $D \alpha=\beta$, and 
let $\gamma$ be any element of $2 \backslash A_{0}$. We consider the irreducible equation connecting $\alpha$ and $\gamma: Q(\alpha, \gamma)=0, Q \in A_{0}\left[t_{1}, t_{2}\right]$. We write $Q^{\prime}$ for the polynomial obtained from $Q$ by applying $D$ to all its coefficients. Then $D \gamma$ can be determined from the equations

$$
\frac{\partial Q}{\partial \alpha} D \alpha+\frac{\partial Q}{\partial \gamma} D \gamma+Q^{\prime}=0
$$

(the polynomial $\partial Q / \partial \gamma$ is prime to $Q$, because $Q$ is irreducible, consequently, $\partial Q / \partial \gamma(\alpha, \gamma) \neq 0$ on $\mathscr{U}$, and therefore, (1.7) always can be solved for $D \gamma)$.

It is easy to verify that this extension has all the properties of a differentiation.

Definition 1. A differential algebraic function field $\left(\mathscr{U}, A_{0}, D\right)$ in one variable is called Fuchsian if all its local rings are closed under differentiation.

We give two criteria for a differential field to be Fuchsian.

Criterion 1. For each element $\alpha \in \mathscr{U}$ we consider the irreducible polynomial connecting $\alpha$ and $D \alpha$ :

(1.8) $\quad Q(D \alpha, \alpha)=Q_{m}(\alpha)(D \alpha)^{m}+\ldots+Q_{0}(\alpha), Q_{j} \in A_{0}\left[t_{1}\right]$.

The differential field is Fuchsian if and only if $\operatorname{deg} Q_{m}=0$ in (1.8) for all $\alpha \in \mathfrak{Q}$.

Proof. Suppose that the field is Fuchsian. Then ord $\alpha \geqslant 0$ implies that $\operatorname{ord}_{V} D \alpha \geqslant 0$. Therefore, by (1.6), $\operatorname{deg} Q_{m}=0$ in (1.8). Now suppose that the field is not Fuchsian. Then there is a local ring $V$ and an element $\alpha \in V$ such that $D \alpha \notin V$, that is, ord $V \alpha \geqslant 0$ and $\operatorname{ord}_{V} D \alpha<0$. Then, by (1.6), $\alpha$ satisfies (1.8) with $\operatorname{deg} Q_{m}>0$.

The following criterion has the advantage that it can be stated in terms of a single element of $\mathfrak{A} \backslash A_{0}$ and is, therefore, handy for verification in practice.

Criterion 2 (the Fuchs conditions). Let $\alpha \in \mathfrak{A}$ be an element such that $\alpha$ and $D \alpha$ generate $\mathfrak{U}$ over $A_{0}$ We consider the irreducible equation (1.8). For the differential field $(\mathfrak{A}, D)$ to be Fuchsian it is necessary and sufficient that the following conditions are satisfied:

A. $\operatorname{deg} Q_{m}=0, \operatorname{deg} Q_{j} \leqslant 2(m-j), 0 \leqslant j \leqslant m$, in (1.8).

B. Suppose that in some local ring $V$

$$
\begin{array}{rll}
D \alpha=b_{0}-t^{n} u_{1}, & b_{0} \in A_{0}, & u_{1} \in U(V), \\
\alpha=a_{0}+t^{m} u_{2}, & a_{0} \in A_{0}, & u_{2} \in U(V),
\end{array}
$$

with $m \geqslant 2$ (such local rings are called branch points over $\alpha$ ). Then $D a_{0}=b_{0}$ and $n \geqslant m-1$.

C. Suppose that at some local ring $V$

$$
\begin{aligned}
D \alpha=t^{-n} u_{1}, & u_{1} \in U(V), \\
\alpha=t^{-m} u_{2}, & u_{2} \in U(V),
\end{aligned}
$$

with $m \geqslant 2$ (such local rings are called ramified poles over $\alpha$ ). Then $n \leqslant m+1$. 
The proof of Criterion 2 is no more difficult that that of Criterion 1 and amounts to differentiation in local rings (see [4], [44]).

Fuchs obtained his Criterion 2 as a necessary and sufficient condition for the general integral of the equation $(0.3)$ to have no movable critical points. Here a critical point of a solution is said to be movable if its position depends on the arbitrary constant in the general integral. In this article we do not make use anywhere of the concepts of general integral and movable critical point, therefore, we do not give rigorous definitions and a statement of the theorem of Fuchs [1], [4]. We remark that condition $\mathrm{C}$ was omitted in Fuchs' original paper. This omission was subsequently repeated in the majority of books on the analytic theory of differential equations (for example, in [4]). The fact that condition $C$ is necessary was first noticed by Hill and Berry in $1910([1], 310 ;[44], 15)$.

One can also examine Fuchsian differential fields when $A_{0}$ is not algebraically closed or has non-zero characteristic. The book [44] is devoted to the general theory of such Fuchsian fields; it carries over to arbitrary Fuchsian fields results obtained in the 19 th century for differential equations without movable critical points.

The importance of Fuchsian differential fields for the analytic theory of differential equations is made clear by the following three results. Classical proofs of these results are contained in [4], Ch. II. For modern proofs and generalizations to arbitrary fields, see [44]. In the statements we give here we assume that $A_{0}$ is algebraically closed and has characteristic 0 .

Theorem 1 (Fuchs). If a Fuchsian differential field (I, $A_{0}, D$ ) has genus 0 , then it is a Riccati field. This means that there is an element $\alpha \in$ I such that $\alpha$ and $D \alpha$ generate $\mathfrak{A}$ over $A_{0}$ and

$$
D \alpha=a_{0}+a_{1} \alpha+a_{2} \alpha^{2}, \quad a_{j} \in A_{0}, \quad 0 \leqslant j \leqslant 2 .
$$

A differential field $\left(\mathfrak{U}, A_{0}, D\right)$ is called a Clairaut field if $\mathbb{A}$ is generated over $A_{0}$ by elements $\alpha$ and $\beta$ such that $D \alpha=0, D \beta=0$.

Theorem 2 (Poincaré). If a Fuchsian differential field ( $\left.\mathfrak{A}, A_{0}, D\right)$ has genus 1 , then it is either a Clairaut field or a Poincaré field. The latter means that $\mathfrak{A}$ is generated over $A_{0}$ by a pair $\alpha$ and $D(\alpha)$ such that

$$
(D(\alpha))^{2}=a\left(\alpha-\lambda_{1}\right)\left(\alpha-\lambda_{2}\right)\left(\alpha-\lambda_{3}\right), \quad a \in A_{0},
$$

$\lambda_{1}, \lambda_{2}, \lambda_{3} \in A_{0}$, the $\lambda_{j}$ are pairwise distinct, and $D\left(\lambda_{i}\right)=0$.

Theorem 3 (Schwarz and Nishioka). Every Fuchsian differential field of genus $\mathrm{g} \geqslant 2$ is a Clairaut field.

Suppose, for example, that $A_{0}$ is the field of all algebraic functions in $z$. The equation $F\left(y^{\prime}, y, z\right)=0$ can be regarded as the equation of a family of curves depending on the parameter $z$. Suppose that $z_{0}$ is a point in general position, that $y_{0}$ and $y_{0}^{\prime}$ are the coordinates of some point on the curve, and that $F\left(y_{0}^{\prime}, y_{0}, z_{0}\right)=0$. It follows from the Fuchs conditions that the Cauchy problem $y\left(z_{0}\right)=y_{0}, y^{\prime}\left(z_{0}\right)=y_{0}^{\prime}$ has a unique solution depending analytically on $y_{0}$ and $y_{0}^{\prime}$. This solution determines an isomorphism between the curves of our family. The classical proofs of Theorems 1,2 , and 3 are based on this argument. For example, to prove Theorem 3 one considers the Weierstrass points that are preserved under the isomorphism.

We remark that the proof of Theorem 3 in $[4], \mathrm{Ch} . \mathrm{II}, \S 14$, is incomplete. 


\section{§2. Admissible solutions}

1. In the preceding section we have described fields connected with differential equations. We now pass to properties of the fields in which we consider solutions of the equations.

Suppose that $f \in A$. We assume that $f$ is continued to a finitely-valued analytic function in C. Suppose that $r>0$ is chosen so that none of the branch points of $f$ project into the set $\{z:|z|=r, z \neq r\}$. Over this set $f$ splits into single-valued branches $f_{j}(j=1, \ldots, k)$. We set

$$
\begin{aligned}
& m\left(r, f_{j}\right)=\frac{1}{2 \pi} \int_{0}^{2 \pi} \log ^{+}\left|f\left(r e^{i \theta}\right)\right| d \theta \quad\left(a^{+}=\max (a, 0)\right), \\
& m(r, f)=\frac{1}{k} \sum_{j=1}^{k} m\left(r, f_{j}\right) .
\end{aligned}
$$

It is easy to show that the function $m(r, f)$ thus defined can be extended by continuity to all values of $r>0$. It is called the Nevanlinna proximity function.

We consider the germ $\nu$ of $f$ over an arbitrary point $z_{0} \in \mathbf{C}$. It can be given by a series

$$
\sum_{j=0}^{\infty} a_{j}\left(z-z_{0}\right)^{(j+k) / n}, \quad a_{j} \in \mathbf{C}, \quad k \in \mathbf{Z}, \quad n \in \mathbf{N} .
$$

We assume that the exponents in the terms with $a_{j} \neq 0$ have no common factor. Therefore, the number $k=$ ord $\nu$ is uniquely determined by the germ. (In $\S 1$ a similar notation had a different meaning, but this should not lead to any confusion.) Let $\nu_{j}(j=1, \ldots, l)$ be all the germs of $f$ projecting into the disc $\{z:|z| \leqslant r\}$ such that ord $\nu_{j}<0$. We set

$$
\begin{aligned}
& n(r, f)=\sum_{j=1}^{l}\left(-\operatorname{ord} v_{j}\right), \\
& N(r, f)=\frac{1}{m} \int_{0}^{r}(n(t, f)-n(0, f)) \frac{d t}{t}+\frac{1}{m} n(0, f) \log r, \\
& T(r, f)=m(r, f)+N(r, f) .
\end{aligned}
$$

In the second formula $m$ denotes the number of branches of $f$. The functions $N(r, f)$ and $T(r, f)$ are called, respectively, the number of poles and the Nevanlinna characteristic. We list the chief properties of $m(r, f)$, $N(r, f)$, and $T(r, f)$. Proofs of these properties for meromorphic functions are contained in [3], [8], [16], and [19]. For the case of algebroid functions, see [15], [50]-[52], and [60].

$$
\begin{aligned}
1^{\circ} . T(r, f) \uparrow \infty \text { as } r & \rightarrow \infty . \text { Here } \\
& T(r, f)=O(\log r), \quad r \rightarrow \infty,
\end{aligned}
$$

if and only if $f$ is an algebraic function (over $\mathbf{C}$ ). 


$$
\begin{aligned}
& 2^{\circ} . T(r, f+g) \leqslant T(r, f)+T(r, g)+O(1), \quad r \rightarrow \infty . \\
& 3^{\circ} . T(r, f g) \leqslant T(r, f)+T(r, g) . \\
& \text { 4․ } T\left(r, \frac{1}{f}\right)=T(r, f)+O(1), \quad r \rightarrow \infty
\end{aligned}
$$

is the Jensen formula.

$$
\text { 5. } T\left(r, \frac{1}{f-a}\right)=T(r, f)+O(1), \quad r \rightarrow \infty, a \in \mathrm{C}
$$

is Nevanlinna's first main theorem.

$$
\begin{aligned}
& 6^{\circ} . T\left(r, f^{n}\right)=n T(r, f)+O(1), \quad r \rightarrow \infty, \quad n \in \mathbf{N} . \\
& 7^{\circ} . m\left(r, \frac{f^{\prime}}{f}\right)=o(T(r, f))+O(\log r), \quad r \rightarrow \infty,
\end{aligned}
$$

outside a set of values of $r$ of finite measure is the Nevanlinna lemma on the logarithmic derivative. This lemma is a fundamental tool in the investigation of meromorphic solutions of differential equations.

$8^{\circ}$. Suppose that $f, g_{1}, \ldots, g_{n}$ are algebroid functions, and that $F \in \mathbf{C}\left[t_{0}, t_{1}, \ldots, t_{n}\right]$ is a polynomial such that $\partial F / \partial t_{0} \neq 0$. Then from $F\left(f, g_{1}, \ldots, g_{n}\right) \equiv 0$ it follows that

$$
T(r, f)=O\left(T\left(r, g_{1}\right)+\ldots+T\left(r, g_{n}\right)\right), \quad r \rightarrow \infty .
$$

More precise estimates are obtained in [13].

All the properties except $4^{\circ}, 5^{\circ}$, and $7^{\circ}$ follow easily from the definition of the characteristic. If $a \in \overline{\mathbf{C}}$, we set

$$
N(r, a, f)=N\left(r, \frac{1}{f-a}\right), a \neq \infty ; \quad N(r, \infty, f)=N(r, f) .
$$

It follows from $5^{\circ}$ that $N(r, a, f) \leqslant T(r, f)+O(1)$, as $r \rightarrow \infty$, for any $a \in \overline{\mathbf{C}}$. The following theorem is often useful.

Valiron's theorem. For all $a \in \overline{\mathbf{C}}$, excluding an exceptional set of plane measure zero,

$$
N(r, a, f)=(1+o(1)) T(r, f), \quad r \rightarrow \infty .
$$

(See, for example, [8], 151.) The estimate of the exceptional set in this theorem can be considerably improved ([16], 280).

2. The Nevanlinna characteristic is convenient for selecting subfields of $A$. We consider a function $\varphi$ on $[0, \infty)$ with the property that $\varphi>0, \log r=$ $=O(\varphi(r))$, as $r \rightarrow \infty$. We write $A_{\varphi}$ for the set of elements of $A$ for which

$$
T(r, f) \approx O(\varphi(r)), \quad r \rightarrow \infty .
$$

Here and in what follows the symbol $\approx$ means that equality holds outside some set of finite measure. This definition is required because later on we have to make use of the lemma on the logarithmic derivative. It follows from $2^{\circ}, 3^{\circ}$, and $4^{\circ}$ that $A_{\varphi}$ is a field, and from $8^{\circ}$ that this field is algebraically closed. If $\varphi(r) \equiv \log r$, then, by $1^{\circ}, A_{\varphi}$ is the field of all algebraic functions over $\mathbf{C}$. We now write $\mathscr{P}_{\varphi}$ for the smallest field that contains $A_{\varphi}$ and all meromorphic functions. Roughly speaking, $\mathscr{P}_{\varphi}$ consists 
of all algebroid functions that have few branch points. For example, if $\varphi(r) \equiv \log r$, then $\mathscr{T}_{\varphi}$ consists of the algebroid functions with finitely many branch points.

We are interested in relations of the form

$$
F\left(x_{x} y\right)=0_{\text {w }} \quad F \in A_{\text {q }}\left[t_{1}, t_{2}\right],
$$

where $x, y \in \mathscr{P}_{\Phi} \backslash A_{\Phi}$. We assume that $F$ is irreducible. We consider the algebraic function field $\mathscr{I}$ in one variable over $A_{\varphi}: \mathfrak{U}=A_{\varphi}\left(t_{1}, t_{2}\right) / F$. If $x, y \in \mathscr{P}_{\varphi} \backslash A_{\varphi}$, then $x$ and $y$ are transcendental over $A_{\varphi}$. The relation (2.1) enables us to define a field homomorphism $\mu: \mathfrak{A} \rightarrow \mathscr{P}_{\varphi}$ that is the identity on $A_{\varphi}$. Under this map, an element $\alpha=R\left(t_{1}, t_{2}\right) \in \mathfrak{A}$ goes tc $R(x, y) \in A_{\varphi}$. The converse is also true: to every field homomorphism $\mu: \Re \rightarrow T_{\varphi}$ that is the identity on $A_{\varphi}$ there corresponds a relation (2.1).

Definition 2. A homomorphism $\mu: \mathfrak{A} \rightarrow \mathscr{P}_{\varphi}$ is called admissible if $\mu$ is the identity on $A_{\varphi}$.

Since any non-zero homomorphism of fields is injective, $\mu(\mathfrak{A}) \not \subset A_{\varphi}$ for an admissible homomorphism. II can be identified with some subfield of $\mathfrak{P}_{\varphi}$. Henceforth we do not distinguish between $\alpha$ and $\mu(\alpha)$ if $\mu$ is an admissible homomorphism.

3. We consider the differential equation

$$
F\left(y^{\prime}, y\right)=0, \quad F \in A_{\varphi}\left[t_{1}, t_{2}\right]
$$

$F$ is irreducible.

Definition 3. A solution $y \in \mathscr{P}_{\varphi}$ of (2.2) is called admissible if $y \notin A_{\varphi}$.

The field $\mathscr{P}_{\varphi}$ is endowed with a natural differentiation $\frac{d}{d z}$. It is clear that the differential equation (2.2) has an admissible solution if and only if there is an admissible homomorphism of differential fields $\mu:(\Re, D) \rightarrow\left(\mathscr{P}_{\varphi}, \frac{d}{d z}\right)$,

$$
\begin{aligned}
& \mathfrak{U}=A_{\varphi}\left(t_{1}, t_{2}\right) / F, \quad F(D \alpha, \alpha)=0, \\
& \mu(D \gamma)=\frac{d}{d z} \mu(\gamma) \text { for all } \gamma \in \mathfrak{U} .
\end{aligned}
$$

4. We now show how Nevanlinna theory can be applied in investigating admissible solutions of differential equations.

First of all we establish the following relation for functions in $\mathfrak{P}_{\varphi}$ :

$$
T\left(r, f^{\prime}\right) \leqslant(2+o(1)) \mathrm{T}(r, f)+O(\varphi(r)), \quad r \rightarrow \infty,
$$

outside some set of finite measure. For this purpose we first estimate $N\left(r, f^{\prime}\right)$. Clearly, $n\left(r, f^{\prime}\right) \leqslant 2 n(r, f)+e(r, f)$, where $e(r, f)$ denotes the number of branch points of $f$ that project into the disc $\{z:|z| \leqslant r\}$. Now $f$ has the form $R\left(h_{1}, \ldots, h_{k}\right)$, where the $h_{j}$ are meromorphic, and $R \in$ $\in A_{\varphi}\left(t_{1}, \ldots, t_{k}\right)$. The branch points of $f$ are, therefore, the branch points of the coefficients of $R$. Consequently,

$$
\int_{0}^{r}(e(t, f)-e(0, f)) \frac{d t}{t}+e(0, f) \log r \approx O(\varphi(r)),
$$


and hence,

(2.4)

$$
N\left(r, f^{\prime}\right) \leqslant 2 N(r, f)+O(\varphi(r)), \quad r \rightarrow \infty,
$$

outside a set of finite measure.

By the lemma on the logarithmic derivative,

$m\left(r, f^{\prime}\right)=m\left(r, \frac{f^{\prime}}{f} \cdot f\right) \leqslant m\left(r, \frac{f^{\prime}}{f}\right)+$

$$
+m(r, f)+O(1) \approx m(r, f)+o(T(r, f))+O(\log r) .
$$

From this and (2.4) we obtain (2.3).

Using only the properties $2^{\circ}, 3^{\circ}, 4^{\circ}$, and $6^{\circ}$ of the Nevanlinna characteristic, A.Z. Mokhon'ko [12] has proved the following theorem:

Suppose that $f$ is a meromorphic function and $R \in A_{\varphi}\left(t_{1}\right)$. Then

$$
T(r, R(f)) \approx n T(r, f)+O(\varphi(r))
$$

where $n$ is the degree of the rational function $R$ (we have made an insignificant change in the statement).

The first theorem of this type was proved by Valiron [60] (see also [2]), for the case when $R$ is a rational function of $f$ and $z$.

We now prove Malmquist's Theorem $I$ in the form given by A.Z. and V.D. Mokhon'ko [14] and Laine [38].

Theorem 4. Suppose that the differential equation $y^{\prime}=R(y), R \in A_{\varphi}\left(t_{1}\right)$, has an admissible solution. Then $R$ is a polynomial of degree at most 2 .

Proof. By the change $y \mapsto y^{-1}+a, a \in \mathrm{C}$, we can put the equation into the form $y^{\prime}=P(y) / Q(y)$, where $P, Q \in A_{\varphi}\left[t_{1}\right], P$ and $Q$ are relatively prime, and $\operatorname{deg} P=2+\operatorname{deg} Q$. Therefore, $n=\operatorname{deg}(P / Q)=2+\operatorname{deg} Q$. Using (2.3) and (2.5) we obtain

$$
(2+\operatorname{deg} Q) T(r, y)+O(\varphi(r)) \approx T\left(r, y^{\prime}\right) \leqslant(2+o(1)) T(r, y)+O(\varphi(r))
$$

as $r \rightarrow \infty$, outside a set of finite measure. Hence it is clear that if $y$ is an admissible solution, then $\operatorname{deg} Q=0$ and $y^{\prime}=P(y)$ is a Riccati equation. Since under a fractional-linear change of the unknown a Riccati equation goes to an equation of the same type, the theorem is proved.

5. Our proof of Malmquist's Theorem II and generalizations of it is based on the same idea. We wish to obtain a relation between the Nevanlinna characteristics for functions $x, y \in \Im_{4}$ connected by (2.1). To do this we suppose that we are given an admissible embedding of $\mathfrak{⿰}=A_{\varphi}\left(t_{1}, t_{2}\right) / F$ into $\mathscr{P}_{4}$. We recall that we identify elements $\alpha \in \mathscr{U}$ with their images under this embedding. By property $8^{\circ}$ of the characteristic, for any $\alpha, \beta \in \Re \backslash A_{\varphi}$, there is a constant $k$ such that

$$
T(r, \alpha) \leqslant k T(r, \beta)+O(\varphi(r)), \quad r \rightarrow \infty,
$$

outside a set of finite measure. We introduce an equivalence relation on the set of all functions defined on $[0, \infty)$ (not necessarily positive): $\psi_{1} \sim \psi_{2}$ if 
for any $\alpha \in \mathfrak{A} \backslash A_{\mathscr{D}}$

$$
\psi_{1}(r)-\psi_{2}(r) \approx o(T(r, \alpha))+O(\varphi(r)), \quad r \rightarrow \infty .
$$

It follows from (2.6) that the words "for any" in this definition can be replaced by "there is". In connection with this definition we write $\psi_{1} \gg 0$ if $\psi_{1} \geqslant \psi_{2}$ for some function $\psi_{2} \sim 0$, and $\psi_{1} \gg \psi_{2}$ if $\psi_{1}-\psi_{2} \gg 0$.

Introducing the notation $q^{+}=\max (q, 0), q^{-}=(-q)^{+}$, we can write any divisor $\delta$ in $\mathfrak{X}$ in the form $\delta=\delta^{+}-\delta^{-}$, where $\delta^{+}$and $\delta^{-}$are non-negative divisors.

Theorem 5. Suppose that $\mathscr{A}$ is admissibly embedded in ${ }_{\varphi}$. Then for any $\alpha, \beta \in \mathfrak{U}$

$$
\operatorname{deg} \delta^{-}(\alpha) T(r, \beta) \sim \operatorname{deg} \delta^{-}(\beta) T(r, \alpha) .
$$

Remark. This theorem and its proof remain valid if $\mathfrak{T}_{\varphi}$ in the statement is replaced by the whole of $A$. The exceptional sets of finite measure also play no part in Theorem 5. We have stated the theorem in a form convenient for subsequent applications.

Suppose, for example, that $\mathfrak{U}$ is a field of genus 0 . Then there is an element $\gamma \in \mathfrak{U}$ such that $\mathfrak{A}=A_{\varphi}(\gamma)$. If $\mathfrak{A}$ is admissibly embedded in $\mathscr{P}_{\varphi}$, Theorem 5 gives

$$
T(r, \alpha) \approx(\operatorname{deg} R+o(1)) T(r, \gamma)+O(\varphi(r)),
$$

where $\alpha=R(\gamma), R \in A_{\varphi}\left(t_{1}\right)$. This is a little weaker than (2.5), but it is enough for our purposes.

It is not known whether $\rho_{\varphi}$ has a subfield of transcendence degree 1 and genus $g \geqslant 2$ over $A_{\varphi}$. If $A_{\varphi}=\mathrm{C}$, then $\mathscr{P}_{\varphi}$ is simply the field of meromorphic functions, and there are no such subfields, by virtue of Picard's theorem that curves of genus $g \geqslant 2$ cannot be uniformized by means of meromorphic functions. If $\varphi=\log r$, then the question reduces to the following: can two transcendental meromorphic functions $f$ and $g$ satisfy a relation

$$
F(f(z), g(z), z) \equiv 0,
$$

where $F$ is a polynomial in three variables such that for a point $z_{0}$ in general position the curve $F\left(x, y, z_{0}\right)=0$ has genus greater than 1 ?

\section{§3. Proof of Theorem 5}

1. Let $f$ be an algebroid function, and let $f_{j}(z), 1 \leqslant j \leqslant n$, denote all the values of this function at a point $z \in \mathbf{C}$. We set $f^{*}(z)=\max _{j}\left|f_{j}(z)\right|$. The function $f^{*}$ is single-valued, non-negative, and continuous in the extended sense. We write $E$ for the smallest class of functions $\sigma: \mathbf{C} \rightarrow \mathbf{R}^{+} \cup\{\{\infty\}$ that contains all functions of the form $f^{*}, f \in A_{\varphi}$, and is closed with respect to addition, multiplication, and taking the upper envelope of finite families. For functions $\sigma \in E$ one can introduce analogues of the Nevanlinna characteristics. We set

$$
m(r, \sigma)=\frac{1}{2 \pi} \int_{0}^{2 \pi} \log ^{+} \sigma\left(r e^{i \theta}\right) d \theta .
$$


It is clear that $m(r, \sigma) \approx O(\varphi(r))$ for $\sigma \in E$. Functions in $E$ can become infinite at isolated points. In a neighbourhood of any such point $z_{0}$

$$
\sigma\left(z-z_{0}\right)\left|z-z_{0}\right|^{k} \rightarrow C, \quad z \rightarrow z_{0},
$$

where $k$ is a rational number and $0<C<\infty$. We call $k$ the order of the pole of $\sigma$ at $z_{0}$. Using this definition of order, we introduce quantities $n(r, \sigma)$ and $N(r, \sigma)$ in the same way as for algebroid functions. It is easy to see that $N(r, \sigma) \approx O(\varphi(r))$ for $\sigma \in E$.

If $f, g \in A$ and $\sigma_{1}, \sigma_{2} \in E$, then the inequality

$$
|f(z)| \leqslant \sigma_{1}(z)|g(z)|+\sigma_{2}(z)
$$

implies that $T(r, f) \leqslant T(r, g)+O(\varphi(r))$ outside a set of finite measure.

Indeed, for example,

$$
N(r, f) \leqslant N(r, g)+N\left(r, \sigma_{1}\right)+N\left(r, \sigma_{2}\right) \approx N(r, g)+O(\varphi(r)),
$$

and $m(r, f)$ can be estimated in a similar way. In particular, for an algebroid function $f$ the conditions $f \in A_{\varphi}$ and $f^{*} \in E$ are equivalent.

Lemma 1. Let $R \in A_{\varphi}\left(t_{1}\right)$ be a rational function such that the degree of its numerator does not exceed that of the denominator, and suppose that $\beta \in A$. Then there is a function $\sigma \in E$ such that from $|\beta(z)| \geqslant \sigma(z)$ it follows that $|R(\beta)(z)| \leqslant \sigma(z)$.

Proof. Let

$$
\begin{gathered}
R(\beta)=\frac{a_{m} \beta^{m}+\ldots+a_{0}}{b_{m} \beta^{m}+\ldots+b_{0}}=\frac{a_{m}+\ldots+a_{0} \beta^{-m}}{b_{m}+\ldots+b_{0} \beta^{-m}}, \\
a_{j}, \quad b_{j} \in A_{\varphi}, \quad 0 \leqslant j \leqslant m, \quad b_{m} \neq 0 .
\end{gathered}
$$

If

then

$$
|\beta(z)| \geqslant \frac{2}{\left|b_{m}(z)\right|}\left(\sum_{j=0}^{m-1}\left(\left|b_{j}(z)\right|+\left|a_{j}(z)\right|\right)+1\right)^{\text {def }}=s_{1}(z)
$$

$$
|R(\beta)(z)| \leqslant 2 \frac{\left|a_{m}(z)\right|}{\left|b_{m}(z)\right|}+1 \stackrel{\text { det }}{=} s_{2}(z) .
$$

There is a function $\sigma \in E$ such that $s_{1}(z) \leqslant \sigma(z)$ and $s_{2}(z) \leqslant \sigma(z)$. This implies what the lemma asserts.

Lemma 2. Suppose that $\alpha, \beta \in \mathfrak{A}$ and $\delta^{-}(\alpha)=\delta^{-}(\beta)$; then $T(r, \alpha) \approx$ $\approx T(r, \beta)+O(\varphi(r))$.

Proof. We consider the relation between $\alpha$ and $\beta$ :

$$
Q_{m}(\beta) \alpha^{m}+\ldots+Q_{0}(\beta)=0, \quad Q_{j} \in A_{\varphi}\left[t_{1}\right], \quad 0 \leqslant j \leqslant m .
$$

By the hypothesis of the lemma, from ord $\beta<0$ it follows that $\operatorname{ord}_{V} \alpha=$ $=\operatorname{ord}_{V} \beta$, consequently, the Newton diagram is a segment with the endpoints $\left(m, \operatorname{deg} Q_{m}\right)$ and $\left(0, m+\operatorname{deg} Q_{m}\right)$. Therefore, in (3.1)

$$
\begin{aligned}
& \operatorname{deg} Q_{0}=m+\operatorname{deg} Q_{m}, \\
& \operatorname{deg} Q_{j} \leqslant m-j+\operatorname{deg} Q_{m}, \quad 1 \leqslant j \leqslant m-1 .
\end{aligned}
$$


We rewrite (3.1) in the form

$$
\begin{gathered}
\left(\frac{\beta}{\alpha}\right)^{m}+R_{m-1}(\beta)\left(\frac{\beta}{\alpha}\right)^{m-1}+\ldots+R_{0}(\beta)=0, \\
R_{j} \in A_{\varphi}\left(t_{1}\right), \quad 0 \leqslant j \leqslant m-1 .
\end{gathered}
$$

From (3.2) it follows that the $R_{j}$ satisfy the conditions of Lemma 1.

Applying the standard estimate for the roots of a polynomial in terms of its coefficients, we find that there is a function $\sigma \in E$ such that $|\beta(z)| \geqslant \sigma(z)$ implies that $|\beta(z) / \alpha(z)| \leqslant \sigma(z)$. Consequently, we always have $|\beta(z)| \leqslant$ $\leqslant \sigma(z)(|\alpha(z)|+1)$. Hence, $T(r, \beta) \leqslant T(r, \alpha)+O(\varphi(r))$ outside a set of finite measure. Interchanging $\alpha$ and $\beta$, we obtain the assertion of the lemma.

Lemma 3. Suppose that $\alpha, \beta, \gamma \in \mathfrak{A}$, that the divisors $\delta^{-}(\alpha)$ and $\delta^{-}(\beta)$ do not intersect (that is, $\left.\delta^{-}(\alpha) \delta^{-}(\beta)=0\right)$, and that $\delta^{-}(\alpha)+\delta^{-}(\beta)=\delta^{-}(\gamma)$. Then $T(r, \gamma) \approx T(r, \alpha)+T(r, \beta)+O(\varphi(r))$.

Proof. We consider again the relation (3.1) between $\alpha$ and $\beta$. By the hypothesis of the lemma, from ord $\beta<0$ it follows that $\operatorname{ord}_{V} \alpha \geqslant 0$. Therefore, the Newton diagram has no sections with a negative gradient, and so in (3.1)

$$
\operatorname{deg} Q_{j} \leqslant \operatorname{deg} Q_{m} \quad(j=0, \ldots, m-1) .
$$

Now (3.1) can be rewritten in the form

$$
\alpha^{m}+R_{m-1}(\beta) \alpha^{m-1}+\ldots+R_{0}(\beta)=0
$$

where by (3.4) the rational functions $R_{j}$ satisfy the conditions of Lemma 1. Hence we find that there is a function $\sigma_{1} \in E$ such that from $|\beta(z)| \geqslant \sigma_{1}(z)$ it follows that $|\alpha(z)| \leqslant \sigma_{1}(z)$. Similarly, there is a function $\sigma_{2} \in E$ such that from $|\alpha(z)|>\sigma_{2}(z)$ it follows that $|\beta(z)| \leqslant \sigma_{2}(z)$. We set $\sigma=\max \left(\sigma_{1}, \sigma_{2}\right)$ and show that

$$
T(r, \alpha)+T(r, \beta) \approx T(r, \alpha+\beta)+O(\varphi(r)) .
$$

We write $S_{1}=\{z:|\alpha(z)| \geqslant \sigma(z)\}, S_{2}=\{z:|\beta(z)| \geqslant \sigma(z)\}$. The sets $S_{1}$ and $S_{2}$ do not intersect. We write $N(r, S, f)$ for the numerical function in which we count only those poles that lie in $S$. Then

$$
\begin{aligned}
& N\left(r, S_{1}, \alpha\right) \approx N(r, \alpha)+O(\varphi(r)), \\
& N\left(r, S_{2}, \beta\right) \approx N(r, \beta)+O(\varphi(r)),
\end{aligned}
$$

$$
\begin{aligned}
& N(r, \alpha+\beta) \approx N\left(r, S_{1}, \alpha+\beta\right)+N\left(r, S_{2}, \alpha+\beta\right)+O(\varphi(r)) \approx \\
& \quad \approx N\left(r, S_{1,} \alpha\right)+N\left(r_{2} S_{2}, \beta\right)+O(\varphi(r)) \approx N(r, \alpha)+N(r, \beta)+O(\varphi(r)) .
\end{aligned}
$$

Similarly it can be proved that $m(r, \alpha+\beta) \approx m(r, \alpha)+m(r, \beta)+O(\varphi(r))$, from which we obtain (3.5).

We now remark that $\delta^{-}(\gamma)=\delta^{-}(\alpha+\beta)$. The assertion of the lemma therefore follows from (3.5) and Lemma 2 .

2. Proof of Theorem 5. In $\S 2.5$ we introduced an equivalence relation on the set of all functions on $[0, \infty)$. We write $\mathfrak{T}$ for the corresponding set of classes. We recall that $\mathfrak{T}$ is also equipped with the ordering $\gg$. 
On divisors of the form $\delta^{-}(\alpha), \alpha \in \mathfrak{A}^{\prime}$, we define a function $x\left(\delta^{-}(\alpha)\right)=$ $=T(\alpha)=T(r, \alpha)$. This function takes values in $\mathfrak{T}$ and is well-defined by virtue of Lemma 2. Our aim is to prove that the divisor function $x\left(\delta^{-}(\alpha)\right)$ is "proportional" to deg $\delta^{-}(\alpha)$. The proof consists of several steps. To begin with we extend $x$ to all divisors by linearity so that it becomes a nonnegative class function.

First of all, we remark that $x$ is homogeneous in the following sense: if $\delta^{-}(\alpha)=n \delta^{-}(\beta)$, then $x^{-}\left(\delta^{-}(\alpha)\right) \sim n x\left(\delta^{-}(\beta)\right)$. It is enough to verify this for natural numbers $n$. But $\delta^{-}\left(\beta^{n}\right)=n \delta^{-}(\beta)=\delta^{-}(\alpha)$ and $T\left(\beta^{n}\right) \sim n T(\beta)$ by $\S 2.1,6^{\circ}$.

In order to extend $x$ to all non-negative divisors we use the Riemann-Roch theorem ( $\S 1.3)$. We consider a non-negative divisor $\delta$ that takes a value $k \geqslant 2 g$ at a single point $V$ and is equal to 0 at all other points (we write this divisor in the form $\delta=k V$ ). We claim that there is an element $\alpha \in \mathfrak{A}$ such that $\delta^{-}(\alpha)=\delta$. For if there were no such element in $\mathcal{L}(\delta)$, then we would have $\mathcal{L}(k V)=((k-1) V)$, which is impossible, because by the Riemann-Roch theorem $\operatorname{dim} \mathcal{L}((k-1) V)=k+g-1$, whereas $\operatorname{dim} \mathcal{L}((k-1) V)=k+g-2$. Thus, if the degree of a divisor $\delta$ is sufficiently large, then there is an element $\alpha \in \mathfrak{M}$ such that $\delta^{-}(\alpha)=\delta$. Suppose now that $\delta$ is an arbitrary non-negative divisor. By the above, $n \delta=\delta^{-}(\alpha)$ for some $n \in \mathbf{N}$ and $\alpha \in \mathfrak{x}$. We can, therefore, set $x(\delta)=n^{-1} T(\alpha)$. By homogeneity, as already proved, this is well-defined.

We extend our function $x$ to all divisors of $\mathscr{U}$ in the following way: $x(\delta)=x\left(\delta^{+}\right)-x\left(\delta^{-}\right)$. Clearly,

$$
x(\delta) \gg 0, \quad \text { if } \delta \geqslant 0
$$

It now follows easily from Lemma 3 that

$$
x\left(\delta_{1}+\delta_{2}\right) \sim x\left(\delta_{1}\right)+x\left(\delta_{2}\right) .
$$

For, suppose that $\delta_{j}=\sum_{i} k_{j}^{i} V_{i}$, where the $V_{i}$ are points, $1 \leqslant j \leqslant 3, \delta_{1}+\delta_{2}=$ $=\delta_{3}$, and $k_{1}^{i}+k_{2}^{i}=k_{3}^{i}$. By homogeneity and Lemma 3 we find that $\varkappa\left(\delta_{j}\right)=\sum_{i} k_{j}^{i} \varkappa\left(V_{i}\right)(j=1,2,3)$, which implies (3.7).

The last property of $x$ we used consists in the fact that it is a class function:

$$
x(\delta(\alpha)) \sim 0 \quad \text { for all } \alpha \in \mathfrak{X},
$$

which follows readily from $\$ 2.1,4^{\circ}$. Indeed,

$$
\begin{aligned}
x(\delta(\alpha)) \sim x\left(\delta^{+}(\alpha)\right)-x\left(\delta^{-}(\alpha)\right) \sim x\left(\delta^{-}\left(\frac{1}{\alpha}\right)\right) & - \\
& -x\left(\delta^{-}(\alpha)\right) \sim T\left(\frac{1}{\alpha}\right)-T(\alpha) \sim 0 .
\end{aligned}
$$

3. We can now complete the proof of Theorem 5 . It is enough to prove that if $\operatorname{deg} \delta=0$, then $x(\delta) \sim 0$. Suppose that $\operatorname{deg} \delta=0$. We claim that

$$
x(\delta) \gg 0 \text {. }
$$


Suppose that (3.9) does not hold. This means that there is an element $\alpha \in \mathfrak{N} \backslash A_{\varphi}$, a natural number $n$, and a set $X \subset[0, \infty)$ of finite measure such that simultaneously

$$
\begin{gathered}
x(r, \delta) \leqslant-\frac{1}{n} x\left(r, \delta^{-}(\alpha)\right), \quad r \in X, \\
\varphi(r)=o(x(r, \delta)), \quad r \in X .
\end{gathered}
$$

We consider the divisor $\delta_{1}=(n+1) \delta+\delta^{-}(\alpha)$. Since deg $\delta_{1}=\operatorname{deg} \delta^{-}(\alpha)>0$, by the Riemann-Roch theorem there are a $k \in \mathbf{N}$ and a $\beta \in \mathscr{U} \backslash A_{\varphi}$ such that $k \delta_{1} \geqslant \delta(\beta)$, that is, $k \delta_{1}-\delta(\beta) \geqslant 0$. Applying (3.6), (3.7), and (3.8) in succession, we find that $x\left(k \delta_{1}\right) \gg 0, x\left(\delta_{1}\right) \gg 0, x(\delta) \gg-(n+1)^{-1} x\left(\delta^{-}(\alpha)\right)$, that is,

$$
x(r, \delta) \geqslant-\frac{1+o(1)}{n+1} \times\left(r, \delta^{-}(\alpha)\right)+O(\varphi(r))
$$

outside a set of finite measure; but this contradicts (3.10) and (3.11) and proves (3.9). To obtain the reverse inequality it is sufficient to apply (3.9) to the divisor $-\delta$.

The theorem is now proved.

4. Corollary of Theorem 5. Let $F \in A_{\varphi}\left[t_{1}, t_{2}\right]$ be an irreducible polynomial. We write $m$ and $n$ for its degrees in $t_{1}$ and $t_{2}$, respectively. If the identity $F(f, g)=0$ holds, where $f, g \in \mathscr{P}_{\varphi}$, then

$$
m T(r, f) \approx(n+o(1)) T(r, g)+O(\varphi(r)) .
$$

This is also true for arbitrary algebroid functions $f, g \in A$. See also [70], where estimates are obtained for the non-analytic branches of algebroid functions.

\section{§4. Malmquist's theorem}

1. Theorem 6. Let $\varphi$ be a positive function such that $\log r=O(\varphi(r))$, and let

$$
F\left(y^{\prime}, y\right)=0, \quad F \in A_{\mathrm{q}}\left[t_{1}, t_{2}\right],
$$

be an irreducible differential equation. If (4.1) has an admissible solution, then this equation satisfies the Fuchs conditions.

Proof. Suppose that (4.1) has an admissible solution. Then we can consider the admissible embedding of the corresponding differential field ( $\mathscr{P}, D$ ) in $\left(\mathscr{S}_{\mathfrak{q}}, \frac{a}{d z}\right)$, as was done in $\S 2.3$. Any element $\alpha \in \mathfrak{U} \backslash A_{\leftarrow}$ satisfies an irreducible differential equation

$$
\begin{gathered}
Q_{m}(\alpha)(D \alpha)^{m}+\ldots+Q_{0}(\alpha)=0 \\
Q_{j} \in A_{p_{p}}\left[t_{1}\right], \quad \operatorname{deg} Q_{m}=n .
\end{gathered}
$$

By the change $\alpha \mapsto \alpha^{-1}+a, a \in \mathbf{C}$, in (4.2), we can achieve that

$$
\operatorname{deg} Q_{j}=n+2(m-j) \quad(j=0, \ldots, m) .
$$


We assume that (4.3) holds for (4.2). We search for a relation between $\operatorname{deg} \delta^{-}(\alpha)$ and deg $\delta^{-}(D \alpha)$. By (4.3), the Newton diagram consists of a single segment with gradient -2 . Therefore, if $\operatorname{ord}_{V} \alpha<0$, then $\operatorname{ord}_{V} D \alpha=2 \operatorname{ord}_{V} \alpha$. Moreover, there are $n$ points at which $\operatorname{ord}_{V} \alpha \geqslant 0$ and $\operatorname{ord}_{V} D \alpha<0$ (see 1.6). Thus,

$$
\operatorname{deg} \delta^{-}(D \alpha)=2 \operatorname{deg} \delta^{-}(\alpha)+n .
$$

By Theorem 5,

$$
T(r, D \alpha) \sim\left(2+n\left(\operatorname{deg} \delta^{-}(\alpha)\right)^{-1}\right) T(r, \alpha) .
$$

On the other hand, by $(2.3), T(r, D \alpha) \leqslant(2+o(1)) T(r, \alpha)+O(\varphi(r))$ outside a set of finite measure. Consequently, $n=0$, because $\alpha \in A_{\varphi}$. Thus, the criterion 1 for Fuchsian fields is satisfied and we have proved the theorem.

2. Malmquist's Theorem II is obtained from Theorem 6 if we put $\varphi(r) \equiv \log r$. Then $A_{\varphi}=A_{\log }$ is the field of all algebraic functions over C. If the polynomial in Theorem 6 is linear in $y^{\prime}$, we obtain Theorem 4 . It is worth mentioning especially a slight generalization of Theorem 4 , which we obtain with the aid of Theorems 6 and 1:

Suppose that (4.1) has an admissible solution and that the field $\mathfrak{U}=A_{\varphi}\left(t_{1}, t_{2}\right) / F$ has genus 0 . Then (4.1) can be transformed to a Riccati equation $w^{\prime}=a w^{2}+b w+c, a, b, c \in A_{\varphi}$, by means of an algebraic change $Q(w, y)=0, Q \in A_{\varphi}\left[t_{1}, t_{2}\right]$.

Admissible solutions of the Riccati equation have been studied a great deal ([3], [39] , [45], [63], [64]) by methods of the theory of meromorphic functions.

The special case of Theorem 6 when the degree of $F$ in $y^{\prime}$ is at most 2 and $\varphi(r) \equiv \log r$ was proved by Steinmetz [55]. His method does not enable one to consider the case of an arbitrary polynomial $F$.

We remark that the Fuchs conditions are only necessary and not sufficient for an equation to have even one meromorphic solution. In the case of autonomous equations, the Fuchs conditions are necessary and sufficient for the general solution to be meromorphic. But non-autonomous equations, generally speaking, may have fixed singular points in the finite plane. In this context a class of functions discovered by Steinmetz [55] is of interest; their general solution is meromorphic:

$$
\left(w^{\prime}-\dot{b}(z) w\right)^{2}=w(a(z)+c(z) w)^{2},
$$

where $a, b$, and $c$ are polynomials in $z$. The distribution of the values of the solutions of these equations is studied in detail in [55].

It is profitable to consider the following example [57]:

$$
w^{\prime 2}=\frac{9}{4} z\left(w^{2}-1\right) w^{2} .
$$

This equation satisfies the Fuchs conditions. It has $w_{1}=\left(\cos z^{3 / 2}\right)^{-1}$ as a meromorphic solution, and also the many-valued algebroid solution $w_{2}=\left(\sin z^{3 / 2}\right)^{-1}$. 
3. A number of papers, beginning with [67], is devoted to the study of meromorphic solutions of an equation

$$
\left(y^{\prime}\right)^{m}=P(y, z) / Q(y, z),
$$

in which $P$ and $Q$ as polynomials in $y$ are relatively prime. The coefficient field of $P$ and $Q$ is often regarded as being some algebraically not closed field of rational functions or a subfield of $A_{\varphi}$ consisting of meromorphic functions. We remark that whether $P$ and $Q$ are relatively prime does not depend on what field they belong to. This follows from the properties of the resultant of two polynomials.

It is easy to see that if $P$ and $Q$ are relatively prime and $\operatorname{deg} Q \geqslant 1$, then all irreducible factors of the polynomial $Q(y) s^{m}-P(y)$ are of the form $Q_{n}(y) s^{n}+\ldots+Q_{0}(y)$, with $\operatorname{deg} Q_{n} \geqslant 1$. When we now apply to (4.4) Theorem 6 and the criterion 2 of $\S 1$, we obtain the following result: if (4.4) has an admissible solution, then $\operatorname{deg} Q=0$ and $\operatorname{deg} P \leqslant 2 \mathrm{~m}$. This fact was first proved by Yosida for the case $\varphi(r) \equiv \log r$ [67]. Thus, (4.4), if it has an admissible solution, of the form

$$
\left(y^{\prime}\right)^{m}=P(y), \quad P \in A_{\varphi}\left[t_{1}\right], \quad \operatorname{deg} P \leqslant 2 m .
$$

Using criterion 2, we can select among the equations (4.5) those that belong to the Fuchs class (the details of this are carried out in $[1], \S 13.8$ ). One obtains the following types of equations:

A. The Riccati equation $y^{\prime}=a y^{2}+b y+c, a, b, c \in A_{\varphi}$.

B. Equations of the form $\left(y^{\prime}\right)^{m}=a P(y), a \in A_{\varphi}, P \in \mathrm{C}\left[t_{1}\right]$. All such equations can be integrated by means of elliptic functions ([1], \$13.81).

C. The equation $\left(y^{\prime}\right)^{2}=c(z)(y-a(z))(y-b)(y-d), a, c \in A_{\varphi}, b, d \in \mathbf{C}$.

Thus, (4.4), if it has an admissible solution, belongs to one of the types A, B, or C. In the particular case of rational coefficients $(\varphi(r) \equiv \log r)$, this was established independently by Bank and Kaufman [26] and Steinmetz [54].

4. We now pass on to the investigation of properties of the solutions. Up to the present time, a fairly complete investigation of properties of the solutions has only been achieved for the equation (4.4), that is, effectively for the equations A, B, and C. Suppose that the coefficients of these equations are rational functions. Then the order of every transcendental solution is either $n / 2$ or $n / 3$, where $n \geqslant 0$ is an integer. If the order is 0 , then $T(r, y)=(c+o(1)) \log ^{2} r$ [24], [25], [26]. The distribution of the values of meromorphic functions determined by the equations $\mathrm{A}, \mathrm{B}$, and $\mathrm{C}$ is studied in detail in [54], [55], and [57]. In [24] it is conjectured that any transcendental solution of an arbitrary first-order differential equation with rational coefficients has the property that $T(r, y) \neq o\left(\log ^{2} r\right), r \rightarrow \infty$. With the help of Theorems 1, 2, 3, 4, and 6 we can obtain a more precise statement. 
Theorem 7. Suppose that a transcendental meromorphic function y satisfies the equation $F\left(y^{\prime}, y, z\right)=0, F \in \mathbf{C}\left[t_{1}, t_{2}, t_{3}\right]$. Then $y$ is a function of finite rational order $\rho$. If $\rho>0$, then the limit

$$
0<\lim _{r \rightarrow \infty} r^{-\rho} T(r, y)<\infty
$$

exists, and if $\rho=0$, then the limit

$$
0<\lim _{r \rightarrow \infty}\left(\log ^{2} r\right)^{-1} T(r, y)<\infty
$$

exists.

That the order is finite was first proved by Gol'dberg [5] ; see also [71] . (Incidentally, [5] contains the only reference to Malmquist's Theorem II known to the author.)

The following example shows that the case (4.7) can actually occur:

$$
w^{\prime 2}=\left(z^{2}-1\right)^{-1}\left(w-e_{1}\right)\left(w-e_{2}\right)\left(w-e_{3}\right) \text {. }
$$

Here $e_{1}, e_{2}$, and $e_{3}$ are such that the Weierstrass elliptic function 8 with periods $2 \pi i$ and 1 satisfies the equation $\left(8^{\prime}\right)^{2}=\left(8^{\circ}-e_{1}\right) \cdot\left(8-e_{2}\right)\left(8-e_{3}\right)$.

The general solution of $(4.8)$ is given by the formula $w=8\left(\int\left(z^{2}-1\right)^{-1 / 2} d z\right)$. It is not hard to show that $w$ is meromorphic on $\mathbf{C}$ and satisfies (4.7) (see also the following $\S 5$ ). This example was discovered by Bank and Kaufman [24].

\section{§5. The asymptotic behaviour of solutions}

1. In this section we give a sketch of a proof of Theorem 7 and present some related results.

Suppose that a transcendental meromorphic function $y$ satisfies the differential equation $F\left(y^{\prime}, y, z\right)=0$, where $F$ is a polynomial in three variables. As usual, we regard $F$ as a polynomial in two variables over the field $A_{\log }\left(A_{\log }=A_{\varphi}\right.$ for $\left.\varphi(r) \equiv \log r\right)$. Then $y$ must satisfy an irreducible equation over $A_{\mathrm{log}}$

$$
Q\left(y^{\prime}, y\right)=0, \quad Q \in A_{\log }\left[t_{1}, t_{2}\right] .
$$

A transcendental function $y$ satisfying (5.1) gives an admissible embedding of differential fields $\mu:(\mathfrak{A}, D) \rightarrow\left(\mathfrak{P}_{\mathbb{q}}, \frac{d}{d z}\right)$. By Theorem 6, $\mathfrak{T}$ is Fuchsian. By Theorem 3, the genus $g$ of $y$ is 0 or 1 (in the case of larger genus there are no admissible embeddings of $\mathfrak{A}$ in $\mathscr{S}_{\varphi}$ ). We now consider the two cases separately.

2. $g=0$. In this case $\mathfrak{A}$ is a Riccati field, by Theorem 1 , consequently, there is an element $\alpha \in \mathfrak{I}$ satisfying the equation

$$
w^{\prime}=a w^{2}+b w+c, \quad a, b, c \in A_{\log } .
$$


By a linear substitution $w=d x+e, d, e \in A_{\mathrm{log}}$, (5.2) takes the form

$$
x^{\prime}=h(z)-x^{2}, \quad h \in A_{\log } \text {. }
$$

The latter equation in its turn reduces to a linear second-order equation, and the asymptotic behaviour of its solutions is well known. For details, see [3].

3. $g=1$. By Theorem $2, \mathfrak{A}$ is a Poincare field, that is, some element $w \in$ satisfies the equation

$$
\begin{gathered}
\left(w^{\prime}\right)^{2}=a(z)\left(4 w^{3}-g_{2} w-g_{3}\right), \quad g_{2}, g_{3} \in \mathbf{C}, \\
g_{2}^{3}-27 g_{3}^{2} \neq 0,
\end{gathered}
$$

where $a(z)$ is an algebraic function. We remark that by Theorem 5 it is sufficient to prove (4.6) or (4.7) for any $\alpha \in \backslash A_{\log }$ in place of $y$. It is, therefore, sufficient to study the asymptotic behaviour of solutions of (5.3). The general solution of this equation has the form $w=8(\Phi(z))$, where the Weierstrass elliptic function 8 satisfies the equation $\left(8^{\circ}\right)^{2}=48^{3}-g_{2} 8^{\circ}-g_{3}$, and $\Phi(z)$ is an Abelian in tegral, $d \Phi / d z=(a(z))^{-1}$.

Suppose that $r_{0}>0$ is so large that for $|z|>r_{0}$ the Abelian integral has no branch points with finite projections. We fix an arbitrary connected piece of the Riemann surface of $\Phi$ over $\left\{z:|z|>r_{0}\right\}$ and we call the restriction of $\Phi$ to this piece a branch of the Abelian integral over $\infty$. For any such branch one of the two asymptotic equations holds:

$$
\Phi(z)=a z^{\lambda}+o\left(|z|^{\lambda}\right), \quad z \rightarrow \infty, \quad a \in \mathbf{C},
$$

where $\lambda$ is a rational number, or

$$
\Phi(z)=a \log z+b+o(1), \quad z \rightarrow \infty, \quad a, b \in \mathbf{C} .
$$

It is clear that if there are branches of the form (5.4), then only those for which $\lambda>0$ have an influence on the asymptotic behaviour of $w$. Let $\lambda_{0}$ be maximal among the numbers $\lambda$ corresponding to the branches (5.4), and suppose that $\lambda_{0}>0$. It is not hard to show that then

$$
T(r, w)=(\text { const }+o(1)) r^{2 \lambda_{0}}, \quad r \rightarrow \infty .
$$

4. We consider in more detail the case when there are no branches of the form (5.4) with $\lambda>0$. Then there must be at least one branch of the form (5.5) (otherwise $w$ would have an essential singularity at $\infty$ ). We consider any branch of (5.5). Without loss of generality, we may assume that $a=1$ and $b=0$ in (5.5), since under a linear change of its argument 8 goes to the same function with another period lattice. The function $\Phi$ is single-valued on the Riemann surface of the logarithm in a neighbourhood of $\infty$, and

$$
\Phi(z)=\log z+o(1), \quad z \rightarrow \infty .
$$

It is clear that $w$ has no branch points for $|z|>r_{0}$, since $\Phi$ is holomorphic for such $z$, and $\wp$ is meromorphic on C. Moreover, $w$ is an algebroid function, therefore, $w$ is single-valued on the Riemann surface of $z^{1 / n}$ for some $n \geqslant 1$ and $z \geqslant r_{0}$. We consider the new function

$$
u(z)=w\left(z^{n}\right)=\wp_{1}(E(z))
$$


where $\varangle_{1}$ is the Weierstrass function with a new period lattice, and

$$
E(z)=\log z+o(1), \quad z \rightarrow \infty,
$$

on the Riemann surface of the logarithm. It is clear that $u$ is single-valued. We claim that one of the periods of $\gamma_{1}$ is $2 \pi i$. By (5.6), (5.7), and the fact that $u$ is single-valued, we have the relation

$$
\wp_{1}(\zeta+2 \pi i+\alpha(\zeta))=\wp_{1}(\zeta)
$$

where $\alpha(\zeta)=o(1)$ as $\operatorname{Re}(\zeta) \rightarrow \infty$.

Let $\zeta_{0}$ be arbitrary and let $\zeta_{1}, \ldots, \zeta_{j}, \ldots$ be an infinite sequence of points that are congruent to $\zeta_{0}$ modulo the periods of $\wp_{1}$, as $\operatorname{Re} \zeta_{j} \rightarrow \infty$. Substituting $\zeta_{j}$ in (5.8) and passing to the limit as $j \rightarrow \infty$, we obtain

$$
\wp_{1}\left(\zeta_{0}+2 \pi i\right)=\gamma_{1}\left(\zeta_{0}\right) \text {. }
$$

In view of the fact that $\zeta_{0}$ was arbitrary, $\delta_{1}$ has the period $2 \pi i$.

Let $\tau$ be the second period of $\wp_{1}$. To simplify the ensuing formulae, we suppose that $\tau$ is real. The case of an arbitrary $\tau$ can be examined in exactly the same way.

We consider the half-strip

$$
\Pi=\{\zeta: \operatorname{Re} \zeta>0, \quad 0<\operatorname{Im} \zeta<2 \pi\} .
$$

Let $a \in \mathbf{C}$ be a number such that $\wp_{1}(z) \neq a$ on the boundary of $\Pi$, and let $\left(z_{k}\right)$ be a sequence consisting of all the $a$-points of $\delta_{1}$ in $\Pi$. It is clear that $\operatorname{dist}\left(z_{k}, \partial \Pi\right) \geqslant \delta>0$. Suppose that $r_{0}$ is so large that $|E(z)-\log z| \leqslant \delta / 2<1$ for $|z|>r_{0}$. We consider the image $V(r)$ under $E(z)$ of the annulus $r_{0}<|z|<r$ with a cut along the positive semi-axis. We can choose a branch of $E(z)$ so that

$$
\begin{aligned}
&\left\{\zeta: \log r_{0}+1<\right.\left.\operatorname{Re} \zeta<\log r-1 ; \frac{\delta}{2}<\operatorname{Im} \zeta<2 \pi-\frac{\delta}{2}\right\}= \\
&=\Pi^{\prime}(r) \subset V(r) \subset \Pi^{\prime \prime}(r)= \\
&=\left\{\zeta: \log r_{0}-1<\operatorname{Re} \zeta<\log r+1 ;-\frac{\delta}{2}<\operatorname{Im} \zeta<2 \pi+\frac{\delta}{2}\right\} .
\end{aligned}
$$

Suppose now that $b \in \mathbf{C}$ is so close to $a$ that all $b$-points of $\wp_{1}$ lie in a $\delta / 4$-neighbourhood of $a$-points. Temporarily, we write $n(D, b, f)$ for the collection of $b$-points of $f$ in $D$. Then for all $b$ in the indicated neighbourhood of $a$,

$$
n\left(\Pi^{\prime}(r), b, \wp_{1}\right) \leqslant n(r, b, i)+O(1) \leqslant n\left(\Pi^{\prime \prime}(r), b, \wp_{1}\right)+O(1), \quad r \rightarrow \infty .
$$

Since $\delta_{1}$ has a real period, $n\left(\Pi^{\prime}(r), b, \wp_{1}\right)=n\left(\Pi^{\prime \prime}(r), b, \wp_{1}\right)+O(1)=c \log r+O(1)$,

$$
c=\text { const }, \quad r \rightarrow \infty .
$$

Hence, $n(r, b, u)=c \log r+o(1), r \rightarrow \infty$. Therefore,

$$
N(r, b, u)=\int_{r_{0}}^{r} \frac{n(t, b, u)}{t} d t+O(1)=(c+o(1)) \log ^{2} r, \quad r \rightarrow \infty .
$$


The last equation holds for all $b$ in some neighbourhood of $a$. By Valiron's theorem $(\S 2.1)$ we conclude from this that $T(r, u)=(c+o(1)) \log ^{2} r$. Consequently, $T(r, w)=(c+o(1)) \log ^{2} r$, as required.

5. Theorem 7 does not settle the question of the growth of meromorphic solutions of first-order equations with rational coefficients. It is not known, for example, whether any rational number can serve as the order of a meromorphic solution. The only examples known are when $\rho=n / 2$ or $n / 3$, where $n$ is a non-negative integer [26].

We present some results on the growth of entire solutions that are not contained in Theorem 7.

Theorem 8 (Malmquist). Suppose that $y$ is an entire solution of the equation $F\left(y^{\prime}, y\right)=0$, where $F$ is a polynomial with rational coefficients and irreducible in the algebraically closed field of rational functions $A_{\mathbf{l o g}}$. Then

$$
T(r, y)=(\text { const }+o(1)) r^{n / 2}, \quad r \rightarrow \infty,
$$

where $n$ is a natural number.

This theorem is, in fact, contained in [42] (we have made an insignificant change in the statement).

Theorem 9. Let $F$ be an arbitrary polynomial with rational coefficients. Every entire transcendental solution of the equation $F\left(y^{\prime}, y\right)=0$ is of order not less than $1 / 2$ (see [17], 70).

The papers [5], [68], and [22] contain effective methods for giving upper bounds for a meromorphic solution in terms of the coefficients of the equation.

\section{§6. Higher-order equations}

1. Up to now we have been concerned exclusively with first-order equations. In this section we attempt to apply the theory set out in $\S \S 1-3$ to equations of higher order. For other results on higher-order equations, see $\$ 7$.

We write $H(y)$ for an arbitrary differential polynomial, that is, a si'm of differential monomials $a y^{j_{0}}\left(y^{\prime}\right)^{j_{1}} \ldots\left(y^{(k)}\right)^{j_{k}}$, where $a \in A_{\varphi}$. The numbers $k, d=j_{0}+j_{1}+\ldots+j_{k}$, and $x=j_{0}+2 j_{1}+\ldots+(k+1) j_{k}$ are called, respectively, the order, the degree, and the differential weight of the monomial. The order $k(H)$ of a differential polynomial $H$ is defined as the greatest of the orders of the monomials in $H$. The degree $d(H)$ and the weight $\chi(H)$ are defined in a similar way.

Suppose that $H_{1}$ is a differential polynomial over $A_{\varphi}$. A meromorphic solution of the differential equation $H_{1}(y)$ is said to be admissible if $y \notin A_{\varphi}$.

Theorem 10. Let $F \in A_{\varphi}\left[t_{1}, t_{2}\right]$ be an irreducible polynomial and $H(y)$ an arbitrary differential polynomial over $A_{\varphi}$. If the equation

(6.1) $F(H(y), y)=F_{m}(y) H^{m}(y)+\ldots+F_{0}(y)=0, \quad F_{j} \in A_{\Psi}\left[t_{1}\right]$, has an admissible solution, then $\operatorname{deg} F_{m}=0$. 
Proof. Let $y$ be an admissible solution. Then there is an admissible embedding $\mathfrak{X} \rightarrow \mathfrak{T}_{\varphi}$, where $\mathfrak{A}=A_{\varphi}\left(t_{1}, t_{2}\right) / F$, under which $t_{1}$ goes to $H(y)$ and $t_{2}$ to $y$. As usual, we identify elements with their images. Suppose that $\operatorname{deg} F_{m} \neq 0$. Then by (1.6), there is a local ring $V$ in $\mathfrak{A}$ such that $y \in V$, $H(y) \notin V$. Let $t$ be a generator of the maximal ideal $I$ of $V$. We fix $V$ and $t$ up to the end of the proof.

By the Riemann-Roch theorem, there is an element $w \in \mathfrak{O}$ that belongs to all the local rings of $\mathfrak{I}$ except $V$, and in $V$

$$
w=t^{-q} u_{1}, \quad u_{1} \in U(V), \quad q \in \mathbf{N} .
$$

(We recall that $U=U(V)$ denotes the set of units of $V$.) The following equations hold:

$$
\begin{array}{ll}
P_{r}(w) y^{r}+\ldots+P_{0}(w)=0, & P_{j} \in A_{\mathrm{p}}\left[t_{1}\right], \\
Q_{l}(w) H^{l}+\ldots+Q_{0}(w)=0, & Q_{j} \in A_{\mathrm{p}}\left[t_{1}\right]
\end{array}
$$

(we assume, as always, that (6.3) and (6.4) are irreducible). Since $V$ is the unique point at which ord $V w<0$ and since $\operatorname{ord}_{V} y \geqslant 0$ at this point, the Newton diagram of (6.3) consists of sections with non-negative gradient, therefore,

$$
\operatorname{deg} P_{j} \leqslant \operatorname{deg} P_{T} \quad(i=0, \ldots, r-1) .
$$

Similarly, since ord ${ }_{V} H<0$, the Newton diagram of (6.4) consists only of sections with negative gradient, therefore,

$$
\operatorname{deg} Q_{j}<\operatorname{deg} Q_{0} \quad(j=1, \ldots, l) .
$$

We now consider (6.3) and (6.4) as identities between the algebroid functions $H(y), w$, and $y$. We write $X_{1}$ for the set of projections of all branch points of $H(y)$ and $w$. Now let $X_{2}$ be the set of projections of all zeros and poles of all the coefficients of $P_{j}$ and $Q_{j}$ and the coefficients of $H$. Suppose that $z_{0} \notin X_{1} \cup X_{2}$. If $w$ has a pole at $z_{0}$, then, by (6.3) and (6.5), $y\left(z_{0}\right)$ is finite, and, by (6.4) and $(6.6), H(y)$ has a pole at $z_{0}$. Since this is impossible, all the poles of $w$ are contained in $X_{1} \cup X_{2}$. Hence it is not hard to deduce that

$$
N(r, w) \approx O(\varphi(r))
$$

We now wish to estimate $m(r, w)$. For this we need the following notation. We take any element $x \in \mathfrak{U}$. There is an irreducible relation

$$
S(x, t)=0, \quad S \in A_{\varphi}\left[t_{1}, t_{2}\right] \text {. }
$$

We define the elements $\partial x / \partial t$ and $\partial x / \partial z$ of $\mathscr{A}$ by the formulae

$$
\frac{\partial x}{\partial t}=-\frac{D_{2} S(x, t)}{D_{1} S(x, t)}, \quad \frac{\partial x}{\partial z}=-\frac{S^{\prime}(x, t)}{D_{1} S(x, t)},
$$

where $D_{1}$ and $D_{2}$ are the operators of differentiation of a polynomial with respect to the first and second variable, respectively, and $S^{\prime}$ is the polynomial obtained from $S$ by differentiating its coefficients with respect to $z$. We remark that $\partial x / \partial z$ depends on the choice of $t$, but this does not matter for our purposes, since $t$ is fixed. 
It is easy to see that the decomposition (1.4) at $V$ :

$$
x=a_{0} t^{m}+a_{1} t^{m+1}+\ldots, a_{\jmath} \in A_{\Phi},
$$

can be differentiated according to the usual rules:

$$
\begin{aligned}
& \frac{\partial x}{\partial t}=m a_{0} t^{m-1}+(m+1) a_{1} t^{m}+\ldots, \\
& \frac{\partial x}{\partial z}=\frac{d a_{0}}{d z} t^{m}+\frac{d a_{1}}{d z} t^{m+1}+\ldots
\end{aligned}
$$

Suppose now that

$$
y=t^{n} u_{2}, \quad u_{2} \in U, \quad n \geqslant 0 .
$$

We regard (6.8) as an equation between algebroid functions over $\mathbf{C}$ and differentiate it repeatedly with respect to $z\left({ }^{\prime}=\frac{d}{d z}\right)$ :

$$
\begin{gathered}
y^{\prime}=n t^{n} \frac{t^{\prime}}{t} u_{2}+t^{n+1} \frac{t^{\prime}}{t} \frac{\partial u_{2}}{\partial t}+t^{n} \frac{\partial u_{2}}{\partial z}=t^{n}\left(\frac{t^{\prime}}{t} u_{3}+u_{4}\right), \quad u_{3}, u_{4} \in V ; \\
y^{(j)}=t^{n} Q_{j}\left(\frac{t^{\prime}}{t}, \frac{t^{\prime \prime}}{t}, \ldots, \frac{t^{(j)}}{t}\right), \quad Q_{j} \in V\left[t_{1}, \ldots, t_{j}\right]
\end{gathered}
$$

for any $j \in \mathbf{N}$. We recall that $V\left[t_{1}, \ldots, t_{j}\right]$ is the ring of polynomials with coefficients in $V$. Substituting these expressions in $H(y)=H\left(y, y^{\prime}, \ldots, y^{(k)}\right)$, we obtain

$$
H(y)=t^{n d} P\left(\frac{t^{\prime}}{t}, \ldots, \frac{t^{(k)}}{t}\right), \quad P \in V\left[t_{1}, \ldots, t_{k}\right],
$$

where $d=d(H)$. From (6.2) we find that $t^{-q}=w u_{5}, u_{5} \in U$. Differentiating this equation repeatedly with respect to $z$, we obtain

$$
\text { (6.10) } \frac{t^{(j)}}{t}=M_{j}\left(\frac{w^{\prime}}{w}, \ldots, \frac{w^{(\jmath)}}{w}\right), \quad M_{j} \in V\left[t_{1}, \ldots, t_{j}\right], \quad j \in \mathbf{N} .
$$

Substituting (6.10) in (6.9) and noting that $H=t^{-p} u_{6}, u_{6} \in U$, and $p=-\operatorname{ord}_{V} H>0$, we obtain

$$
t^{-p-n d}=\mathscr{Y}_{1}\left(\frac{w^{\prime}}{w}, \ldots, \frac{w^{(k)}}{w}\right), \quad \mathscr{G}_{1} \in \mathbf{V}\left[t_{1}, \ldots, t_{k}\right],
$$

and finally, by virtue of (6.2),

$$
w^{s}=\mathscr{Y}_{2}\left(\frac{w^{\prime}}{w}, \ldots, \frac{w^{(k)}}{w}\right), \quad \dddot{\mathscr{Y}}_{2} \in V\left[t_{1}, \ldots, t_{k}\right],
$$

with $s>0$.

We now estimate the coefficients of $y_{2}$. If $x \in V$, then the irreducible polynomial

$$
Q_{m}(w) x^{m}+\ldots+Q_{0}(w)=0, \quad Q_{j} \in A_{m}\left[t_{1}\right], \quad 0 \leqslant j \leqslant m,
$$

satisfies the condition $\operatorname{deg} Q_{j} \leqslant \operatorname{deg} Q_{m}$, therefore, we can apply Lemma 1 of $\$ 3$ to the equation

$$
x^{m}+\frac{Q_{m-1}(w)}{Q_{m}(w)} x^{m-1}+\ldots+\frac{Q_{0}(w)}{Q_{m}(w)}=0
$$

Consequently, there is a function $\sigma \in E$ such that $|w(z)| \geqslant \sigma$ implies that $|x(z)| \leqslant \sigma$. Applying this to the coefficients of $\mathscr{G}_{2}$, we obtain from (6.11) the inequality

$$
|w(z)|^{s} \leqslant \sigma_{1}(z)\left(\left|\frac{w^{\prime}(z)}{w(z)}\right|+\ldots+\left|\frac{w^{(k)}(z)}{w(z)}\right|+1\right)^{N},
$$


which is valid for some $\sigma_{1} \in F$ and $N>0$. Hence, by the lemma on the logarithmic derivative, $m(r, w) \approx o(T(r, w))+O(\varphi(r)), r \rightarrow \infty$. Together with (6.7), this shows that $w \in A_{\varphi}$, which contradicts the assumption that the solution is admissible. This proves the theorem.

2. We now consider some consequences of Theorem 10. Suppose that the differential polynomial has the form $H(y)=y^{(k)}$. In the equation

$$
\left(y^{(k)}\right)^{m}+F_{m-1}(y)\left(y^{(k)}\right)^{m-1}+\ldots+F_{0}(y)=0, \quad F_{j} \in A_{\Phi}\left[t_{1}\right],
$$

we make the substitution $y=w^{-1}+a$, where $a \in \mathrm{C}$ is a suitable constant. The equation takes the form

$$
\begin{gathered}
u^{n}(H(w))^{m}+P_{m-1}(u)(H(w))^{m-1}+\ldots+P_{0}(w)=0, \\
P_{j} \in A_{v[}\left[t_{1}\right],
\end{gathered}
$$

where $H$ is some differential polynomial and $n=\max \left(\operatorname{deg} F_{j}-(k+1)(m-j)\right) \geqslant$ $\geqslant 0$. Applying Theorem 10, we obtain the following result: for the irreducible equation

$$
F_{m}(y)\left(y^{(k)}\right)^{m}+\ldots+F_{0}(y)=0, \quad F_{j} \in A_{\Psi}\left[t_{1}\right]
$$

to have an admissible solution it is necessary that

$$
\operatorname{deg} F_{j} \leqslant(k+1)(m-j) \quad(i=0, \ldots, m) .
$$

For $k=1$ this reduces to the condition $A$ of criterion 2 of $\S 1$. It is interesting that (6.13) is the same as the necessary condition given by Chazy [28] for the absence of movable singular points in the general integral of (6.12).

If $F$ in Theorem 10 is linear in $H$, then the equation is

$$
H\left(y^{(k)}, \ldots, y\right)=\frac{P(y)}{Q(y)}, \quad P, Q \in A_{\Psi}\left[t_{1}\right],
$$

to which [30], [40], [58], [59], and [54] are devoted. We assume that $P$ and $Q$ are relatively prime. In [30] and [54] independent proofs are given that for the absence of an admissible solution of (6.14) it is necessary that $\operatorname{deg} Q=0$ and $\operatorname{deg} P \leqslant \varkappa(H)$. Various special cases of this assertion were obtained earlier in [40] and [58]. [59] contains the same result, but with a different definition of an admissible solution.

We deduce the result quoted about equation (6.14) from Theorem 10 and the theorem (2.5) of A.Z. Mikhon'ko. By Theorem 10, deg $Q=0$. On the other hand, it is easy to show that

$$
T(r, H(y)) \leqslant(x(H)+o(1)) T(r, y)+O(\varphi(r))
$$

outside a set of finite measure (this relation is similar to (2.3)). It follows from this and (2.5) that $\operatorname{deg} P \leqslant x(H)$ in (6.14).

We now consider admissible solutions of (6.14) for which

$$
N(r, y) \approx O(\varphi(r)), \quad r \rightarrow \infty,
$$

in particular, entire solutions. If there is an admissible solution, then $\operatorname{deg} Q=0$, by Theorem 10 . Now let $d=d(H)$ be the degree of $H$. 
If $\operatorname{deg} P>d$, then after dividing (6.14) by $y^{d}$, by the lemma on the logarithmic derivative we obtain the estimate

$$
m(r, y) \approx O(\varphi(r)), \quad r \rightarrow \infty
$$

Thus, if $\operatorname{deg} P>d$, then (6.14) has no admissible solutions with the property (6.15). This result was obtained in [66], and for $k=1$ in [67].

3. In the proof of Theorems 6 and 10 , the fact that the relevant meromorphic algebroid functions are defined on the whole plane is used essentially at only one place, in applying the lemma on the logarithmic derivative. This enables us to prove similar theorems for functions that are defined in the unit disc and in a half-plane. Here for the case of the disc we make use of the Nevanlinna characteristic, and for the half-plane we use the Tsudzi characteristic (see [8]). We content ourselves with stating a result similar to Theorem 6 for functions in the unit disc.

The lemma on the logarithmic derivative in this case takes the form

$$
m\left(r, \frac{w^{\prime}}{w}\right) \leqslant(x+1+o(1)) \log (1-r)^{-1}+o(T(r, w)), \quad r \notin X,
$$

where $X \subset[0,1)$ is some set for which

$$
\int_{X}(1-r)^{-x-1} d r<\infty
$$

(see [3] and [16], 256).

Suppose that a positive function $\varphi(r)$ is given on $[0,1)$ with the property that $\log (1-r)^{-1}=o(\varphi(r))$. We write $A_{\varphi}$ for the set of algebroid functions on $\{z:|z|<1\}$ for which

$$
T(r, f)=O(\varphi(r)), \quad r \rightarrow 1, \quad r \notin X,
$$

where $X$ is some set with the property (6.16), for any $x \geqslant 0$.

Theorem 11. If an irreducible equation $F\left(y^{\prime}, y\right)=0, F \in A_{\varphi}\left[t_{1}, t_{2}\right]$, has a solution $y \notin A_{\varphi}$ that is meromorphic on $\{z:|z|<1\}$, then it satisfies the Fuchs conditions.

An analogue of Malmquist's Theorem I for functions that are meromorphic in the unit disc was first obtained by Hille [32] .

We define the order of a function meromorphic in the disc by the formula

$$
\rho=\varlimsup_{r \rightarrow 1} \frac{\log ^{+} T(r, f)}{-\log (1-r)} .
$$

Theorem 12. Suppose that a first-order equation with rational coefficients has a solution that is meromorphic in the unit disc. Then the order of the solution is a finite rational number.

This result follows from Theorem 11 in the same way as Theorem 7 follows from Theorem 6. Earlier, the assertion of Theorem 12 was known only for solutions that are holomorphic in the disc [61]. Theorem 10 also has an analogue for functions that are meromorphic in the disc. 
We do not linger to state corresponding results for a half-plane. Some a priori estimates of the growth of solutions in a half-plane are contained in [7], [18], and [22].

We also mention [72], which studies meromorphic solutions of homogeneous second-order equations. Such equations are reduced to first order by the substitution $w=y^{\prime} / y$, after which one can apply the theory in $\S \S 1-5$.

\section{$\S 7$. Autonomous equations of Briot-Bouquet type}

1. In contrast to first-order equations, the information available at present on meromorphic solutions of higher-order equations is very meagre. The only equations that have been studied with reasonable completeness are certain special equations whose general solution is meromorphic: Painlevé equations (see [1] and [4]). The properties of meromorphic solutions of Painlevé equations are investigated in detail in the classical paper [73].

We consider a very simple class of higher-order equations, for which a complete investigation of its meromorphic solutions is possible in certain cases. These are the equations of Briot-Bouquet type

(7.1) $\quad F\left(y^{(k)}, y\right)=F_{m}(y)\left(y^{(k)}\right)^{m}+\ldots+F_{0}(y)=0, \quad F_{j} \in \mathbf{C}[t]$.

$F$ is an irreducible polynomial. For $k=1$ these are the classical BriotBouquet equations mentioned in the Introduction. If (7.1) with $k=1$ has a non-constant meromorphic solution, then all its solutions are meromorphic. For $k>1$ this is no longer true. Picard [47] has studied meromorphic solutions of (7.1) for $k=2$ (see Theorem 15 below). Even now, 100 years after [47], the equations (7.1) continue to arouse interest [31], [33] - [36], [10], [74]. But it is clear from [33], [35], and [74] that Picard's result has been forgotten.

First of all, we remark that every transcendental solution of (7.1) is admissible, therefore, $(6.13)$ is a necessary condition for the existence of transcendental solutions. We give more precise necessary conditions. For this purpose we consider the Riemann surface $\mathscr{F}$ of the algebroid function $s(y)$ defined by the relation $F(s, y)=0$. We write $M$ for the set of points of this Riemann surface that project to $y=\infty$. For each point $V \in M$ we set $q(V)=\operatorname{ord}_{V} s / \operatorname{ord}_{V} y$.

Theorem 13. Suppose that (7.1) has a transcendental meromorphic solution $y$. Then:

i. $q(V)=1$ for at most two points $V \in M$; for all other points $V \in M$ we have $q(V)=1+k / n$, where $k$ is the order of the equation and $n$ is a natural number.

ii. For $y$ to be entire it is necessary and sufficient that $q(V)=1$ for all $V \in M$.

iii. If $y$ has at least one pole of order $n$, then there is a point $V \in M$ for which $q(V)=1+k / n$. 
iv. If there is a point $V \in M$ such that $q(V)=1+k / n$, then every meromorphic solution has infinitely many poles of order $n$.

The condition (6.13) is obtained from this with the aid of the Newton diagram.

Suppose that (7.1) has a meromorphic solution $y$. Then the pair of functions $y^{(k)}$ and $y$ gives a covering map $\pi: \mathbf{C} \rightarrow \mathscr{F}$. The following theorem holds for $\pi$.

Picard's theorem. Suppose that $\pi$ is non-rational. If the genus of $F$ is 0 , then every point $V \in \mathscr{F}$, with the exception of at most two, has infinitely many inverse images in $\mathbf{C}$. If the genus of $\mathscr{F}$ is 1 , then every point $V \in \mathscr{F}$ has infinitely many inverse images in $\mathbf{C}$. If the genus of $\mathscr{F}$ is greater than 1 , then there are no such maps.

We write $W$ for the set of all elliptic functions and functions of the form $R(\exp a z)$, where $R$ is rational and $a \in \mathbf{C}$.

We claim that for $k=1$ all meromorphic transcendental solutions of (7.1) belong to $W$.

We consider a point $V \in \mathscr{F}$ that is not a branch point and has infinitely many inverse images $z_{1}, z_{2}, \ldots$ under $\pi$. The Cauchy problem for the equation $F\left(y^{\prime}, y\right)=0$ with the initial condition $\pi(0)=V$ has a unique solution, therefore, the meromorphic functions $y\left(z-z_{j}\right)(j=1,2, \ldots)$ coincide. Consequently, $y$ is a periodic function. Without loss of generality we may assume that its period of least modulus is $2 \pi i$. If every point $V \in \mathscr{F}$ has finitely many inverse images in the $\operatorname{strip}\{z: 0<\operatorname{Im} z<2 \pi\}$, then it is easy to see that $y=R(\exp z)$, where $R$ is a rational function. If some point $V \in \mathscr{F}$ has infinitely many inverse images in this strip, then the preceding arguments are again applicable, consequently, $y$ is an elliptic function. This argument seems to be due to Rellich [49], although the assertion we have proved was already known to Briot and Bouquet (see also [74], where another proof is given).

It can happen that a similar argument is applicable to certain equations (7.1) with $k>1$.

Lemma 4. Suppose that $k$ is odd. Then there are only finitely many meromorphic solutions of (7.1) with a pole at 0 .

This lemma is proved by substituting the Laurent series with undetermined coefficients in (7.1) and successively computing these coefficients. Making use of Rellich's idea, Theorem 13, and Lemma 4, we can obtain the following result.

Theorem 14. Suppose that $k$ is odd and (7.1) has a meromorphic solution with at least one pole. Then this solution belongs to $W$.

For a proof, see [10]. 
Lemma 4 is not true for even $k$ (there is a mistake in [36] at this point). But for $k=2$ the following theorem holds:

Theorem 15. If (7.1) with $k=2$ has a meromorphic solution $y$, then $y \in W$. (See [47], and also [33], [35], and [74].)

When the genus of $F$ is 1 , there is a complete description of the meromorphic solutions of (7.1) [10]. In this case, by the uniformization theorem [9], we must have

$$
y^{(k)}=\Phi_{1}(g(z)), \quad y=\Phi_{2}(g(z)),
$$

where $\Phi_{1}$ and $\Phi_{2}$ are elliptic functions and $g$ is an entire function.

Differentiating the second equation in (7.2) $k$ times and substituting the result in the first equation, we obtain the relation

$$
\sum \Phi_{j}(g(z)) h_{j}(z)=0
$$

where the $\Phi_{j}$ are elliptic functions with a common period lattice, and the $h_{j}$ are differential polynomials in $g$. Using Valiron's theorem $(\$ 2.1)$, it can be shown that the equation (7.3) is possible in just one case, when $g=a z+b$. Thus, the only transcendental meromorphic solutions of (7.1) when $\mathscr{F}$ is of genus 1 are the elliptic functions.

This result, and also Theorems 14 and 15, make plausible the conjecture that transcendental meromorphic functions satisfying (7.1) always belong to $\boldsymbol{W}$. But this has not yet been proved even for the equation $y^{\mathrm{IV}}=y^{5}$.

2. Identities like (7.3) arise in other problems of the analytic theory of differential equations. The following interesting result was obtained by Steinmetz in an investigation of the factorization of meromorphic solutions.

Theorem 16. Let $F_{0}, \ldots, F_{m}$, and $h_{0}, \ldots, h_{m}$ be arbitrary meromorphic functions, $m \geqslant 1, F_{j} \neq 0,0 \leqslant j \leqslant m$, and let $g$ be a non-constant entire function such that

$$
\sum_{j=0}^{m} T\left(r, h_{j}\right) \leqslant K T(r, g), \quad K=\text { const. }
$$

If

$$
F_{0}(g) h_{0}+F_{1}(g) h_{1}+\ldots+F_{m}(g) h_{m} \equiv 0,
$$

then there are polynomials $P_{0}, \ldots, P_{m}, P_{j} \neq 0$, such that

$$
P_{0}(g) h_{0}+P_{1}(g) h_{1}+\ldots+P_{m}(g) h_{m} \equiv 0 .
$$

We now consider a differential equation of the form

$$
H(y)=H\left(y^{(k)}, \ldots, y\right)=0,
$$

where $H$ is a differential polynomial over the field of rational functions. From Theorem 16 we can obtain, for example, this result. 
Theorem 17 (Steinmetz [56]). Let $f$ be a transcendental meromorphic function and $g$ an entire function. If the meromorphic function $y=f(g)$ satisfies (7.4), then $f$ and $g$ satisfy, respectively, the equations

$$
H_{1}(f)=0, \quad H_{2}(g)=0,
$$

where $H_{1}$ and $H_{2}$ are differential polynomials with $d\left(H_{1}\right) \leqslant d(H), \varkappa\left(H_{1}\right) \leqslant \varkappa(H)$.

Regarding the factorization of solutions of differential equations, see also [45] and [46].

In this survey we have not touched on questions of the growth of meromorphic solutions of higher-order equations. Results in this area are of an extremely special nature; see, for example, [6] and [23]. In the case of entire solutions, a great deal more has been done; in this case one can apply the Wiman-Valiron theory and the modifications of it set out in detail in [2], [3], [29], and [17].

\section{References}

[1] E.L. Ince, Ordinary differential equations, Longmans, Green and Co., London 1926. MR 6-65.

Translation: Obyknovennye differentsial'nye uravneniya, DNTVU, Kharkov 1939.

[2] G. Valiron, Fonctions analytiques, Presses Universitaires de France, Paris 1954. MR 15-861.

Translation: Analiticheskie funktsii, Gosudarstv. Izdat. Tekhn.-Teor. Lit., Moscow 1957.

[3] H. Wittich, Neuere Untersuchungen über eindeutige analytische Funktionen, Springer-Verlag, Berlin-Heidelberg-New York 1955. MR 17-1067.

Translation: Noveishie issledovaniya po odnoznachnym analiticheskim funktsiyam, Gosudarstv. Izdat. Fiz.-Mat. Lit., Moscow 1960. MR 23 \# A333.

[4] V.V. Golubev, Lektsii po analiticheskoi teorii differentsial'nykh uravnenii (Lectures on the analytic theory of differential equations), second ed., Gosudarstv. Izdat. Tekhn.-Teor. Lit., Moscow-Leningrad 1950. MR 13-131.

[5] A.A. Gol'dberg, On one-valued integrals of differential equations of the first order, Ukrain. Math. Zh. 8 (1956), 254-261. MR 19-32.

[6] - The growth of meromorphic solutions of second-order differential equations, Differentsial'nye Uravneniya 14 (1978), 824-829, 956. MR 58 \# 11412. $=$ Differential Equations 14 (1978), 584-588.

[7] - and A.Z. Mokhon'ko, The rate of growth of the solutions of algebraic differential equations in angular domains, Differentsial'nye Uravneniya 11 (1975), 1568-1574. MR 57 \# 16768.

$=$ Differential Equations 11 (1975), 1169-1173.

[8] - and I.V. Ostrovskii, Raspredelenie znachenii meromorfnykh funktsii (The distribution of values of meromorphic functions), Izdat. Nauka, Moscow 1970. MR 43 \# 6439.

[9] A. Hurwitz and R. Courant, Vorlesungen über allgemeine Funktionentheorie und elliptische Funktionen, mit einen Anhang von H. Röhrl, Springer-Verlag, BerlinHeidelberg-New York 1964. MR 30 \# 3959.

Translation: Teoriya funktsii, Izdat. Nauka, Moscow 1968. 
[10] A.E. Eremenko, Meromorphic solutions of equations of Briot-Bouquet type, Teor. Funktsii, Funktsional Anal. i Prilozhen. (Khar'kov) 1982, no. 38.

[11] S. Lang, Introduction to algebraic and Abelian functions, Addison-Wesley, Reading, MA, 1972. MR 49 \# 8983.

Translation: Vvedenie v algebraicheskie $i$ abelevy funktsii, Mir, Moscow 1976. MR 54 \# 7483.

[12] A.Z. Mikhon'ko, The Nevanlinna characteristics of certain meromorphic functions, Teor. Funktsii, Funktsional Anal. i Prilozhen. (Khar'kov) 1971, no. 14, 83-87. MR 45 \# 7058.

[13] - The Nevanlinna characteristics for a certain class of meromorphic functions, Teor. Funktsii, Funktsional Anal. i Prilozhen. (Khar'kov) 1976, no. 25, 95-105. MR 55 \# 665.

[14] - and V.D. Mokhon'ko, Estimates of the Nevanlinna characteristics of certain classes of meromorphic functions, and their applications to differential equations, Sibirsk. Mat. Zh. 15 (1974), 1305-1322. MR 53 \# 8425. $=$ Siberian Math. J. 15 (1974), $921-934$.

[15] V.D. Mokhon'ko, A lemma on the logarithmic derivative for algebroid functions, Teor. Funktsii, Funktsional Anal. i Prilozhen. (Khar'kov) 1974, no. 20, 112-122. MR 51 \# 13231.

[16] R. Nevanlinna, Eindeutige analytische Funktionen, Springer-Verlag, Berlin 1936. MR 6-59. Translation: Odnoznachnye analiticheskie funktsii, Gosudarstv. Izdat. Tekhn.-Teor. Lit., Moscow-Leningrad 1941.

[17] S.I. Strelitz, Asimptoticheskii svoistva analiticheskikh reshenii differential'nykh uravnenii (Asymptotic properties of analytic solutions of differential equations), Izdat. Mintis, Vilnius 1972. MR 58 \# 17268.

[18] E.D. Fainberg, On solutions of first-order differential equations that are meromorphic in a half-plane, Issled. Perspekt. Problem Peredach. Informatsii, (Khar'kov) 1974, no. 313, 44-49.

[19] W.K. Hayman, Meromorphic functions, Clarendon Press, Oxford 1964. MR 29 \# 1337.

Translation: Meromorfnye funktsii, Mir, Moscow 1966. MR 35 \# 5617.

[20] N.G. Chebotarev, Teoriya algebraicheskikh funktsii (The theory of algebraic functions), Gosudarstv. Izdat. Tekhn.-Teor. Lit., Moscow-Leningrad 1948. MR 10-697.

[21] C. Chevalley, Introduction to the theory of algebraic functions of one variable, Amer. Math. Soc., New York 1951. MR 13-64.

Translation: Vvedenie $v$ teoriyu algebraicheskikh funktsii ot odnoi peremennoi, Gosudarstv. Izdat. Fiz.-Mat. Lit., Moscow 1959.

[22] S.B. Bank, A general theorem concerning the growth of solutions of first-order algebraic differential equations, Compositio Math. 25 (1972), 61-70. MR 47 \# 7093.

[23] — On determining the growth of meromorphic solutions of algebraic differential equations having arbitrary entire coefficients, Nagoya Math. J. 49 (1973), 53-65. MR 47 \# 8939.

[24] and R.P. Kaufman, On meromorphic solutions of first-order differential equations, Comment. Math. Helv. 51 (1976), 289-299. MR 55 \# 3375.

[25] - On the order of growth of meromorphic solutions of firstorder differential equations, Math. Ann. 241 (1979), 57-67. MR 81d:34003. 
[26] S.B. Bank and R.P. Kaufman, On the growth of meromorphic solutions of the differential equation $\left(y^{\prime}\right)^{n}=R(z, y)$, Acta. Math. 144 (1980), 223-248. MR 81k:30028.

[27] L. Bieberbach, Theorie der gewöhnlichen Differentialgleichungen auf funktionentheoretischer Grundlage dargestellt, second ed., Springer-Verlag, Berlin-HeidelbergNew York 1953. MR 15-703.

[28] J. Chazy, Sur les équations différentielles du 3ème ordre et d'ordre súperieur dont l'intégrale générale à ses points critiques est fixe, icta Math. 34 (1911), 317-378.

[29] W.H.J. Fuchs, Asymptotic evaluation of integrals, and Wiman-Valiron theory, Complex analysis and its applications (Lectures, Internat. Sem., Trieste 1975), Vol. I, 235-283, Internat. Atomic Energy Agency, Vienna 1976. MR 58 \# 29706.

[30] F. Gackstatter and I. Laine, Zur Theorie der gewöhnlichen Differentialgleichungen im Komplexen, Annales Polon. Math. 38 (1980), 259-287. MR 82g:34007.

[31] E. Hille, Ordinary differential equations in the complex domain, second ed., WileyInterscience, New York-London 1976. MR 58 \# 17266.

[32] — On some generalizations of the Malmquist theorem, Math. Scand. 39 (1976), 59-79. MR 56 \# 12361.

[33] - Non-linear differential equations: questions and some answers, Differential equations (Proc. Internat. Conf., Uppsala 1977), 101-108, Almqvist and Wiksell, Stockholm 1977. MR 57 \# 16751.

[34] - Remarks on Briot-Bouquet differential equations. I, Comment. Math. (Special Issue) 1 (1978), 119-132. MR 81g:34007; II, J. Math. Anal. Appl. 65 (1978), 572-585. MR 81i:34003.

[35] , Second-order Briot-Bouquet differential equations, Acta Sci. Math. (Szeged) 40 (1978), 63-72. MR 80i:34005.

[36] - Higher order Briot-Bouquet differential equations, Ark. Mat. 16 (1978), 271 -286. MR $81 \mathrm{k}: 34004$.

[37] H.P. Künzi, Sur un théorème de M.J. Malmquist, C.R. Acad. Sci. Paris 242 (1956), 866-868. MR 17-734.

[38] I. Laine, On the behaviour of the solutions of some first-order differential equations, Ann. Acad. Sci. Fenn. Ser. A I, 497 (1971), 1-26. MR 52 \# 8541.

[39] - Admissible solutions of Riccati differential equations, Publ. Univ. Joensuu, Ser. Bl, 1972.

[40] - Admissible solutions of some generalized algebraic differential equations, Publ. Univ. Joensuu, Ser. B10, 1974.

[41] J. Malmquist, Sur les fonctions à un nombre fini de branches satisfaisant à une équation différentielle du premier ordre, Acta Math. 36 (1913), 297-343.

[42] - Sur les fonctions à un nombre fini de branches satisfaisant à une équation différentielle du premier ordre, Acta Math. 42 (1920), 59-79.

[43] —- Sur les fonctions à un nombre fini de branches satisfaisant à une équation différentielle du premier ordre, Acta Math. 74 (1941), 175-196. MR 7-298.

[44] M. Matsuda, First-order algebraic differential equations. A differential algebraic approach, Lecture Notes in Math. 804, 1980. MR 82d:12015.

[45] E. Mues, Über faktorisierbare Lösungen von Riccatischen Differentialgleichungen, Math. Z. 121 (1971), 145-156. MR 44 \# 1806.

[46] — Zur Faktorisierung elliptischer Funktionen, Math. Z. 120 (1971), 157-164. MR 44 \# 5455.

[47] E. Picard, Sur une propriété des fonctions d'une variable et sur une classe d'équations différentielles, C.R. Acad. Sci. Paris 91 (1880), 1058-1060. 
[48] E. Picard, Démonstration d'une théorème générale sur les fonctions uniformes liées par une relation algébrique, Acta. Math. 11 (1887-1888), 1-12.

[49] F. Rellich, Elliptische Funktionen und die ganzen Lösungen von $y^{\prime \prime}=f(y)$, Math. Z. 47 (1940), 153-160. MR 3-241.

[50] H. Selberg, Uber eine Eigenschaft der logarithmischen Ableitung einer meromorphen oder algebroiden Funktion endlicher Ordnung, Ark. Norske Videnskaps-Akademi. I, Math.-Naturvid. Klassa, 1929, no. 14.

[51] — Algebroide Funktionen und Umkehrfunktionen Abelscher Integrale, Ark. Norske Videnskaps-Akademi. I, Math.-Naturvid. Klasse, 1934, no. 8.

[52] - Úber die Werteverteilung der algebroiden Funktionen, Math. Z. 31 (1930), 709-728.

[53] H. Schmidt, Zentralblatt für Math. 27 (1943), 309-311.

[54] N. Steinmetz, Remerkung zu einen Satz von Yosida, Complex analysis, Joensuu 1978 (Proc. Colloq. Univ. Joensuu, Joensuu 1978), 369-377, Lecture Notes in Math. 747 (1979). MR 81a:34007.

[55] —- Ein Malmquistscher Satz für algebraische Differentialgleichungen erster Ordnung, J. Reine Angew. Math. 316 (1980), 44-53. MR $81 \mathrm{~m}: 34007$.

[56] - Über die faktorisierbaren Lösungen gewöhnlicher Differentialgleichungen, Math. Z. 170 (1980), 169-180.

[57] N. Steinmetz, Zur Theorie der binomischen Differentialgleichungen, Math. Ann. 244 (1979), 263-274. MR 81a:34008.

[58] S. Strelitz, A remark on meromorphic solutions of differential equations, Proc. Amer. Math. Soc. 65 (1977), 255-261. MR 58 \# 6427.

[59] - On meromorphic solutions of algebraic differential equations, Trans. Amer. Math. Soc. 258 (1980), 431 -440. MR 82a:34006.

[60] G. Valiron, Sur la derivée des fonctions algébroides, Bull. Soc. Math. France 59 (1931), 17-39.

[61] - Fonctions analytiques et équations différentielles, J. Math. Pures Appl. (9) 31 (1952), 293-303. MR 14-546.

[62] H. Wittich, Ganze transzendente Lösungen algebraischer Differentialgleichungen, Nachr. Akad. Wiss. Göttingen Math.-Phys. K1. 1946, 71 -73. MR 9-145.

[63] - Zur Theorie der Riccatischen Differentialgleichungen, Math. Ann. 127 (1954), 433-440. MR 16-36.

[64] Einige Eigenschaften der Lösungen von $w^{\prime}=a(z)+b(z) w+c(z) w^{2}$, Arch. Math. (Basel) 5 (1954), 226-232. MR 15-862.

[65] C.-C. Yang, A note on Malmquist's theorem on first-order differential equations, Yokohama Math. J. 20 (1972), 115-123. MR 46 \# 9423.

[66] - On meromorphic solutions of generalized algebraic differential equations, Ann. Mat. Pura Appl. (4) 91 (1972), 41-52. MR 47 \# 3679.

[67] K. Yosida, A generalization of Malmquist's theorem, Japan J. Math. 9 (1933), 253-256.

[68] - On a characteristic function of a transcendental meromorphic solution of an algebraic equation of the first order and of the first degree, Proc. Phys.-Math. Soc. Japan 15 (1933), 337-338.

[69] - A note on Malmquist's theorem on first-order algebraic differential equations, Proc. Japan Acad. 53 (1977), 120-123. MR 55 \# 12983.

[70] A.Z. Mokhon'ko, Estimates of the growth of the branches of algebroid functions and of their Nevanlinna characteristics. I, Teoriya Funktsii, Funktional Anal. i Prilozhen. (Khar'kov) 1980, no. 33, 99-107; II, 1981, no. 35, 69-73. MR 82j:30041a, b. 
[71] A.Z. Mokhon'ko, An application of a result of Clunie and Hayman to first-order differential equations, Teoriya Funktsii, Funktsional Anal. i Prilozhen. (Khar'kov) 1981, no. 35, 74-75. MR 82k:34007.

[72] N. Steinmetz, Úber das Anwachsen der Lösungen homogener algebraischer Differentialgleichungen zweiter Ordnung, Manuscripta Math. 32 (1980), 303-308. MR 82d:34010.

[73] P. Boutroux, Recherches sur les transcendentes de M. Painlevé et l'étude asymptotique des équations différentielles du second ordre, Ann. Ecole Normale Superieure 30 (1913), 255-375; 31 (1914), 99-159.

[74] S.B. Bank and R.P. Kaufman, On Briot-Bouquet differential equations and a question of Einar Hille, Math. Z. 177 (1981), 549-559. MR 82g:34006.

Translated by A.J. McIsaac

Low temperature Physical-technical Institute of the USSR Academy of Sciences Received by the Editors 30 April 1981 